MATHEMATICS OF COMPUTATION

Volume 71, Number 237, Pages 77-103

S 0025-5718(01)01304-7

Article electronically published on March 9, 2001

\title{
CONVERGENCE OF AN ITERATIVE ALGORITHM FOR SOLVING HAMILTON-JACOBI TYPE EQUATIONS
}

\author{
JERRY MARKMAN AND I. NORMAN KATZ
}

\begin{abstract}
Solutions of the optimal control and $H_{\infty}$-control problems for nonlinear affine systems can be found by solving Hamilton-Jacobi equations. However, these first order nonlinear partial differential equations can, in general, not be solved analytically. This paper studies the rate of convergence of an iterative algorithm which solves these equations numerically for points near the origin. It is shown that the procedure converges to the stabilizing solution exponentially with respect to the iteration variable. Illustrative examples are presented which confirm the theoretical rate of convergence.
\end{abstract}

\section{INTRODUCTION}

Partial differential equations of the type

$$
p^{T} f(x) \pm p^{T} R(x) p+l(x)=0,
$$

where $x \in \mathbb{R}^{n}, p=V_{x}(x)$ and $R(x), l(x) \geq 0$ are known as Hamilton-Jacobi type equations. They are of considerable importance in the solution of nonlinear optimal control problems [1], nonlinear $H_{\infty}$ problems [2], and in various other areas.

Because (1.1) is a first order nonlinear equation, closed form solutions cannot be found in general. Furthermore, the only information known about $V(x)$ is that it is a $C^{2}$ positive definite solution locally in a neighborhood around the equilibrium point $x=0$ and $V(0)=0$. The size of this neighborhood is not known in advance, and no boundary conditions are known, so traditional numerical methods are not applicable. Approximate solutions of (1.1) have been found either through a power series method [3], [4] or a successive approximation approach [5], [6], [7]. In [8], [9], an iterative procedure was introduced which computes the stabilizing solution of (1.1) for any $x_{0}$ near the origin. By applying the algorithm to sufficiently many points near the origin, an approximate solution over the entire neighborhood

Received by the editor December 1, 1998 and, in revised form, February 17, 2000.

2000 Mathematics Subject Classification. Primary 93B40, 49N35, 65P10.

Key words and phrases. Hamilton-Jacobi equations, convergence, optimal control.

The results reported here are part of the doctoral dissertation of the first author.

This work was supported in part by the National Science Foundation under grant number DMS-9626202 and in part by the Defense Advanced Research Projects Agency (DARPA) and Air Force Research Laboratory, Air Force Materiel Command, USAF, under agreement number F30602-99-2-0551. The U.S. Government is authorized to reproduce and distribute reprints for Governmental purposes notwithstanding any copyright annotation thereon.

The views and conclusions contained herein are those of the authors and should not be interpreted as necessarily representing the official policies or endorsements, either expressed or implied, of the Defense Advanced Research Projects Agency (DARPA), the Air Force Research Laboratory, or the U.S. Government. 
can be pieced together through, for example, polynomial interpolation. Details of implementation and numerical simulations are given in [8], [9].

This paper concentrates on the convergence of the algorithm. After describing the algorithm in Section 2 and proving convergence in Section 3, Section 4 analyzes the rate of convergence to the stabilizing solution of (1.1). It is shown that the procedure converges exponentially with respect to the iteration variable. The rate of convergence bound in the linear case is, however, sharper than that in the nonlinear case. More specifically, if $\lambda_{1}$ is the eigenvalue of an associated linearized Hamiltonian system with the largest negative real part, it is shown that the order of convergence is $0\left(e^{\operatorname{Re} \lambda_{1} t^{i}}\right)$ for nonlinear systems and $0\left(e^{2 \operatorname{Re} \lambda_{1} t^{i}}\right)$ for linear systems, where $t^{i}$ is the time at the $i$ th iteration. Section 5 gives several illustrative examples and studies the rate of convergence in each example. The computed rates are consistent with theoretical predictions.

\section{Algorithm}

2.1. Motivation. First, the problem is formally defined. For the dynamic system

$$
\begin{aligned}
\dot{x} & =f(x)+g(x) u, \\
y & =h(x)
\end{aligned}
$$

with $x \in \mathbb{R}^{n}, u \in \mathbb{R}^{m}, h(x) \in \mathbb{R}^{p}$, where $f(x), g(x), h(x)$ are sufficiently smooth (as needed) and $f(0)=h(0)=0$, a positive semidefinite function $V^{-}(x): U_{\rho} \rightarrow \mathbb{R}$ is sought in an open neighborhood $U_{\rho}$ around the origin which satisfies the HamiltonJacobi equation

$$
H\left(x, V_{x}\right) \equiv V_{x} f(x)+\frac{1}{k} V_{x} g(x) g^{T}(x) V_{x}^{T}+h^{T}(x) h(x)=0
$$

and renders (2.1) asymptotically stable with $u=\frac{2}{k} g^{T}(x) V_{x}^{-}(x)$. Here, $k$ is a parameter which depends on the type of problem being considered. For instance, in a special case of the $H_{\infty}$ problem, $k=4 \gamma^{2}$, where $\gamma$ is the gain, while for optimal control problem $k=-4$. It is assumed throughout this paper that $\sigma(A) \varepsilon C^{-}$, where $A \equiv \frac{\partial f}{\partial x} f(0)$.

The algorithm operates in the Hamiltonian space of (2.2), i.e., it considers the $2 n$-dimensional ODE

$$
\frac{d x}{d t}=H_{p}
$$

$$
\frac{d p}{d t}=-H_{x}
$$

In [10] it is shown that (2.2) has a $C^{2}$ solution $V: U_{p} \rightarrow R$ with $V(0)=V_{x}(0)=0$ if and only if the manifold

$$
M=\left\{(x, p) \varepsilon U_{\rho} \times R^{n}: p=V_{x}^{T}(x)\right\}
$$

is an invariant manifold of the corresponding Hamiltonian system (2.3). In particular if $V(x)$ is a solution of (2.2), then the restriction of the Hamiltonian system to $M$ is

$$
\dot{x}=f(x)+\frac{2}{k} g(x) g^{T}(x), V_{x}^{T}(x)
$$


and

$$
u^{*}(x)=\frac{2}{k} g^{T}(x) V^{T}(x)
$$

is an optimal control for (2.1) if the performance index is taken as $h^{T}(x) h(x)$. Thus, solutions of the Hamilton-Jacobi equation (2.2) can be characterized as invariant manifolds of the equilibrium point $(0,0)$ of the Hamiltonian system (2.3). The manifold corresponding to $u^{*}(x)$, which asymptotically stabilizes (2.1) is called the stable manifold of the Hamiltonian system (2.3).

Furthermore, under suitable conditions for optimal control and $H_{\infty}$ problems, the Hamiltonian system is restricted to the hyperbolic case, i.e., the linearization of (2.3) has no eigenvalues on the imaginary axis. This insures the existence of an $n$-dimensional stable manifold. Finally, for $x$ near the origin, the stable manifold can be expressed as a graph of $x$. Therefore, seeking the stabilizing solution of (2.2) at a fixed point near the origin is equivalent to identifying the stable invariant manifold of (2.3) at that point.

In [14] van der Schaft established the connection, which we use here, between stable manifolds of the Hamiltonian system (2.3) and solutions to the HamiltonJacobi equation (2.1). The purpose of this paper is to provide a numerical algorithm with an associated rate of convergence to compute points on the stable manifold of (2.3) and thereby solve (1.1).

Define

$$
\begin{aligned}
z\left(t, z_{0}\right) & \triangleq\left(\begin{array}{l}
x\left(t, x_{0}, p_{0}\right) \\
p\left(t, x_{0}, p_{0}\right)
\end{array}\right), \\
z_{0} & \triangleq\left(\begin{array}{l}
x_{0} \\
p_{0}
\end{array}\right),
\end{aligned}
$$

where $x\left(t, x_{0}, p_{0}\right)$ and $p\left(t, x_{0}, p_{0}\right)$ are the solutions of (2.3) at time $t$ with initial conditions $x_{0}$ and $p_{0}$. Also define $\bar{H}$ as the linearization of (2.3) around the equilibrium point $(x, p)^{T}=(0,0)^{T}$. Then (2.3) can be rewritten as

$$
\begin{aligned}
& \dot{z}=\bar{H} z+h(z), \quad h \in O\left(\|z\|^{2}\right), \\
& \bar{H}=\frac{\partial}{\partial z}\left(\begin{array}{c}
H_{p} \\
-H_{x}
\end{array}\right)_{z=0}=\left(\begin{array}{cc}
A & \frac{1}{2 k} R \\
-2 Q & -A^{T}
\end{array}\right),
\end{aligned}
$$

where $A=\frac{\partial f}{\partial x}(0), B=g(0), C=\frac{\partial h}{\partial x}(0), R=B B^{T}, Q=C^{T} C$.

Fix a point $x=x_{0}, x_{0} \in U_{\rho}$. Finding the stabilizing solution of (2.2) at $x_{0}$ defined as $p_{0}^{*} \triangleq V_{x}^{-}\left(x_{0}\right)$, is equivalent to finding the vector $p_{0}$ which causes $z\left(t, z_{0}\right) \rightarrow 0$ as $t \rightarrow \infty$.

The algorithm uses this asymptotic behavior of the stabilizing solution of (2.2) to find $p_{0}^{*}$. For a fixed time $t$, it evaluates each possible solution vector $p$ by measuring how close the trajectory of the Hamiltonian system (2.3) passing through $\left(x_{0}, p\right)$ comes to the origin. This is repeated for larger and larger times to produce a sequence of vectors which converge to a point on the stable manifold.

Define the distance function

$$
F\left(t, p_{0}\right) \triangleq\left\|z\left(t, z_{0}\right)\right\|^{2}=\left\|x\left(t, x_{0}, p_{0}\right)\right\|^{2}+\left\|p\left(t, x_{0}, p_{0}\right)\right\|^{2} .
$$

$F$ measures the distance from the solution of (2.6),$z\left(t, z_{0}\right)$, to the origin at time $t$ with initial condition $z_{0}$. Because $x_{0}$ is fixed, $F$ depends solely on the terminal time $t$ and the initial condition $p_{0}$. 
Since the solution of (2.2), $p_{0}^{*}=V_{x}^{-}\left(x_{0}\right)$, lies on the stable manifold of (2.6), $\lim _{t \rightarrow \infty} F\left(t, x_{0}, p_{0}^{*}\right) \rightarrow 0$. Because $F$ is a positive semidefinite function, finding $p_{0}^{*}$, or where the stable manifold intersects $x=x_{0}$, is equivalent to solving

$$
\inf _{p_{0}} \lim _{t \rightarrow \infty} F\left(t, x_{0}, p_{0}\right) \text {. }
$$

However, because the Hamiltonian system (2.6) is nonlinear, a closed form solution for $z\left(t, z_{0}\right)$ is generally not available, and the minimization cannot be done analytically. To approximate a solution numerically, a sequence of times which monotonically increases toward infinity is chosen, and for each element in this sequence $F$ is minimized with respect to $p_{0}$. For each $i=1,2,3, \ldots$ define

$$
\begin{aligned}
t^{i} & \uparrow \infty \\
F^{i} & \triangleq \min _{p_{0}} F\left(t^{i}, p_{0}\right), \\
p_{0}^{i} & \triangleq \operatorname{argmin}_{p_{0}} F\left(t^{i}, p_{0}\right) .
\end{aligned}
$$

As $i$ becomes larger, $t^{i} \rightarrow \infty$, and $F$ can be considered, in a sense, as measuring the asymptotic behavior of candidate solutions. It will be shown in Section 3 that $F^{i} \rightarrow 0$ and $p_{0}^{i} \rightarrow p_{0}^{*}$. Details of the minimization procedure in (2.9b) and (2.9c) are given in $[9$.

2.2. Initial guess. In order to solve (2.9b) and (2.9c) for fixed $i$, an iterative procedure is, in general, required. The procedure we use is described in detail in [8], 9]. For each $i$, a starting estimate is taken to be $p_{0}^{i-1}$. A good estimate for $p_{0}^{*}$ which is taken to be $p_{0}^{0}$ can be found by considering the linearization of (2.1). For any linear system, the solution of the corresponding Hamilton-Jacobi equation is $V^{L}(x)=x^{T} P x$, where $P$ is the stabilizing solution of the Algebraic Ricatti Equation

$$
P A+A^{T} P+\frac{1}{k} P B B^{T} P+C^{T} C=0 .
$$

Thus, the initial guess for the algorithm is

$$
V_{x}^{L}\left(x_{0}\right)=p_{0}^{L}=2 x_{0}^{T} P .
$$

This has a nice geometric interpretation because $p_{0}^{L}$ is the intersection of the stable eigenspace of $\bar{H}$ with the linear variety $x=x_{0}$. Because $x_{0}$ is always chosen near the origin, $p_{0}^{L}$ will lie close to $p_{0}^{*}$.

\section{Convergence}

$p_{0}^{L}$ is the intersection of the stable eigenspace of $H$ with the linear variety $x=x_{0}$. $p_{0}^{L}$ can be found directly from the solution of the Algebraic Ricatti Equation (2.10) and is used as the initial guess in the iteration formulas defined by the algorithm. Because $x_{0}$ is chosen to be near the origin, at $x_{0}$ the stable manifold of (2.3) should be "close" to the stable eigenspace, i.e., $p_{0}^{*}$ should lie near $p_{0}^{L}$. Therefore, instead of considering all vectors $p$ as candidates to lie on the stable manifold, the algorithm considers the set $P^{\prime} \triangleq\left\{p:\left\|p-p_{0}^{L}\right\| \leq \delta\right\}$ for $\delta$ suitably large. See Figure 3.1.

Assumption 1. The point $\left(x_{0}, p_{0}^{*}\right)$ lies in $P^{\prime}$. Since the stable eigenspace is tangent to the stable manifold at 0 , this can be accomplished by choosing $\left\|x_{0}\right\|$ sufficiently small. 


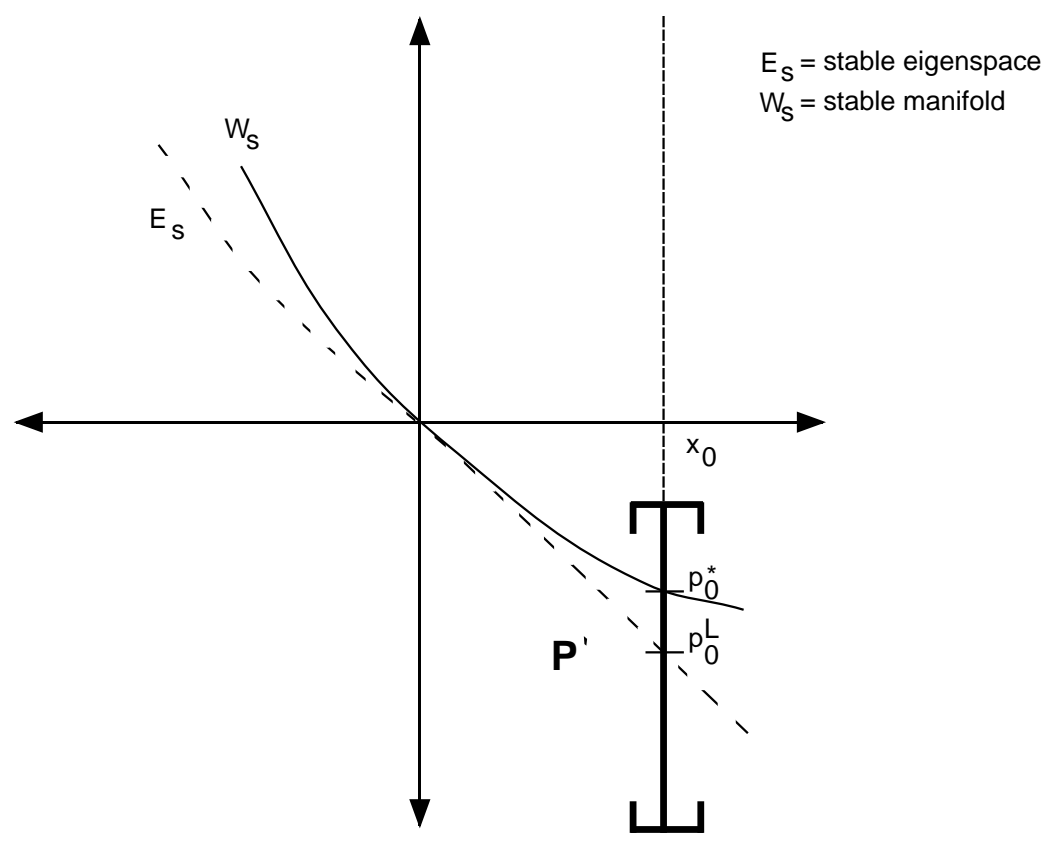

Figure 3.1. Restricting the search space of $p_{0}^{*}$

Assumption 2. For any sequence $\left(t^{j}, x_{0}, p_{0}^{j}\right)$ and $\bar{p}=\lim _{j \rightarrow \infty} p_{0}^{j}$, the following holds:

$$
z\left(\lim _{j \rightarrow \infty} t^{j}, x_{0}, p_{0}^{j}\right)=\lim _{t^{j} \rightarrow \infty} z\left(t^{j}, x_{0}, \bar{p}\right) .
$$

This assumption will prevent situations such as that depicted in Figure 3.2. In the figure as $t^{j} \rightarrow \infty$, there is a sequence $\left(x_{0}, p_{0}^{j}\right)$ with $z\left(t^{j}, x_{0}, p_{0}^{j}\right) \rightarrow 0$. However $p_{0}^{j} \rightarrow \bar{p}$ with $z\left(t^{j}, x_{0}, \bar{p}\right) \rightarrow z^{*}$ as $t^{j} \rightarrow \infty$. Again, this assumption is satisfied when $\left\|x_{0}\right\|$ is sufficiently small since then $p_{0}^{0}=p_{0}^{L}$ and subsequent iterates $p_{0}^{j}$ are close to $p_{0}^{*}$, the point on the stabilizing manifold.

Lemma 3.1. $F^{i} \rightarrow 0$, as $t^{i} \rightarrow \infty$.

Proof. $p_{0}^{*}$ lies on the stable manifold of (2.3), so $\lim _{t^{i} \rightarrow \infty}\left\|z\left(t^{i}, x_{0}, p_{0}^{*}\right)\right\| \rightarrow 0$. For each $t^{i}$,

$$
F^{i}=\left\|z\left(t^{i}, x_{0}, p_{0}^{i}\right)\right\|^{2}=\min _{p_{0} \in P}\left\|z\left(t^{i}, x_{0}, p_{0}\right)\right\|^{2} \leq\left\|z\left(t^{i}, x_{0}, p_{0}^{*}\right)\right\|^{2} .
$$

Therefore, as $t^{i} \rightarrow \infty$,

$$
0 \leq \lim F^{i} \leq \lim \left\|z\left(t^{i}, x_{0}, p_{0}^{*}\right)\right\|^{2}=0 .
$$

That is, $F^{i} \rightarrow 0$.

Because $p_{0}^{i}$ lies in the closed bounded set $P$, it has an accumulation point $\bar{p}$ in $P$. The next lemma states that any accumulation point must lie on the stable manifold of (2.3).

Lemma 3.2. If $F^{i} \rightarrow 0$, then any accumulation point of $p_{0}^{i}$ lies on the stable manifold. Furthermore, there exists exactly one accumulation point, i.e., $p_{0}^{i}$ is a convergent sequence. 


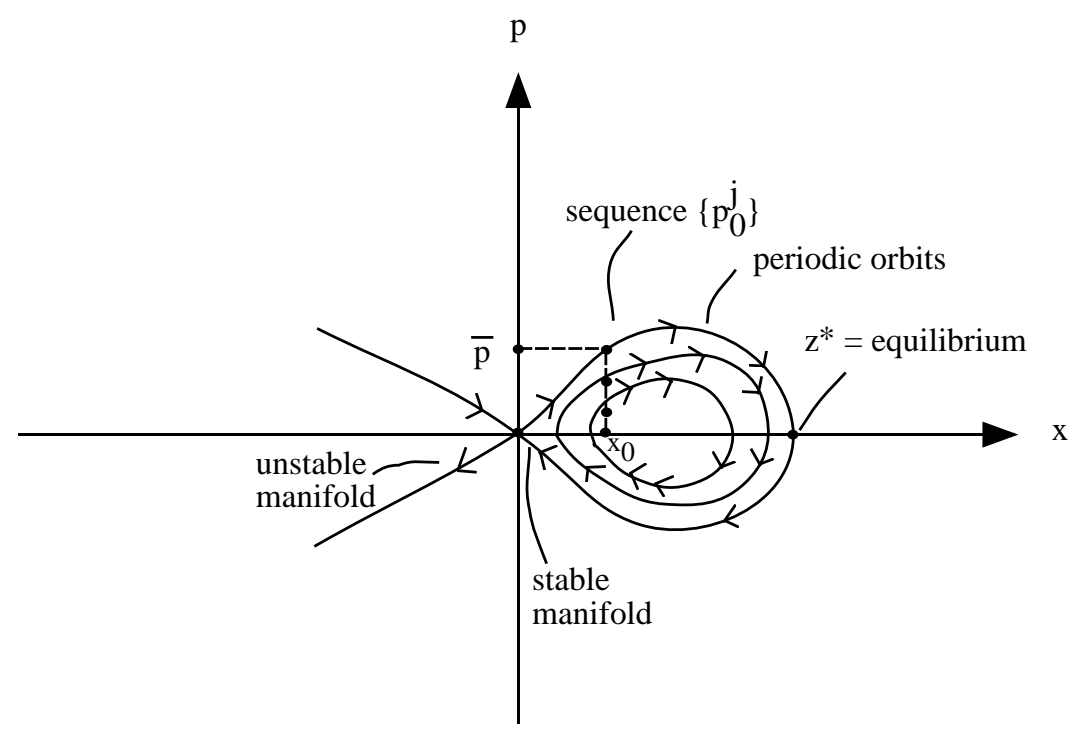

Figure 3.2. A possible phase portrait

Proof. Define $p_{0}^{j}$ as a subsequence of $p_{0}^{i}$ which converges to an accumulation point $\bar{p}$. Then, by assumption 2 ,

$$
\begin{aligned}
\lim _{j \rightarrow \infty}\left\|z\left(t^{j}, x_{0}, p_{0}^{j}\right)\right\| & =\left\|\lim _{j \rightarrow \infty} z\left(t^{j}, x_{0}, p_{0}^{j}\right)\right\| \\
& =\left\|z\left(\lim _{j \rightarrow \infty} t^{j}, x_{0}, \lim _{j \rightarrow \infty} p_{0}^{j}\right)\right\| \\
& =\lim _{t \rightarrow \infty}\left\|z\left(t, x_{0}, \bar{p}\right)\right\|,
\end{aligned}
$$

which goes to zero from Lemma 3.1. Therefore, $\bar{p}$ must lie on the stable manifold.

Now suppose two accumulation points $\bar{p}_{1}, \bar{p}_{2}$ exist. Both points must lie on the stable manifold, but $x_{0}$ was chosen to lie inside the neighborhood where the stable manifold can be written as a graph, i.e., $M^{-} \triangleq p=V_{x}^{-}\left(x_{0}\right)$. Therefore, $\bar{p}_{1}=\bar{p}_{2}=p^{*}$, and the entire sequence converges to the stable manifold.

Together, the above insure convergence of the algorithm to the stabilizing solution of (2.3). We now study the rate of convergence.

\section{RATE of CONVERGENCE}

4.1. Nonlinear systems. Only the case where the Hamiltonian system has simple eigenvalues is considered here. Similar results can be derived when there are multiple eigenvalues. The main result in this section is

$$
\left\|p_{0}^{i}-p_{0}^{*}\right\|=0\left(e^{\left(\operatorname{Re} \lambda_{1}\right) t^{i}}\right),
$$

where $\lambda_{1}$ is the eigenvalue of $\bar{H}$ with the largest negative real part.

A procedure different from (2.9) is given in [2, Appendix B, Section 4] for computing a point on the stable manifold corresponding to $x_{0}$, and also in [15, Section 2.6, Exercise 2.6.7]. In this procedure a two point boundary value problem is formulated for (2.3) with $x(0)=x_{0}$ and $p\left(t^{i}\right)=\xi$, a constant (see [2] B. 73]). Then let $t^{i} \rightarrow \infty$. In [2, B. 80], it is shown that the procedure converges with rate of 
convergence $e^{-\beta^{\prime} t^{i}}$, where $\beta^{\prime}<\beta$ (see [2, B. 46]). $\beta$ corresponds to $\left(-\operatorname{Re} \lambda_{1}\right)$ in our notation, so that the convergence result in [2] is somewhat weaker than ours. In our algorithm given in (2.9), the need to solve a two point boundary value problem is replaced by the minimization problem in (2.9b) and (2.9c).

Since $\bar{H}$ has $2 n$ distinct eigenvalues, there exists a nonsingular transformation matrix $T$ which transforms (2.2) into a diagonalized system, i.e., $T: u \rightarrow z$, or $\left(\begin{array}{l}w \\ v\end{array}\right) \rightarrow\left(\begin{array}{l}x \\ p\end{array}\right)$. Thus, without loss of generality, the Hamiltonian system (2.3) can be written as

$$
\begin{gathered}
\dot{w}=\Lambda w+g_{1}(w, v), \\
\dot{v}=-\Lambda v+g_{2}(w, v),
\end{gathered}
$$

where $\Lambda$ is $\operatorname{diag}\left(\lambda_{1}, \ldots, \lambda_{n}\right)$, and $\operatorname{Re} \lambda_{n} \leq \operatorname{Re} \lambda_{n-1} \leq \cdots \leq \operatorname{Re} \lambda_{1}<0$. We assume that for $\|u\|$ sufficiently small, and $i=1,2$

$$
\left\|g_{i}(u)\right\| \leq K\|u\|^{2}, \quad\left\|\frac{\partial g_{i}}{\partial u}(u)\right\| \leq K\|u\|
$$

Divide $T$ into four $n \times n$ submatrices, i.e., $T \triangleq\left(\begin{array}{cc}\alpha_{1} & \alpha_{2} \\ \alpha_{3} & \alpha_{4}\end{array}\right)$. Then, in the new coordinates, the algorithm consists of the sequence of problems

$$
\begin{gathered}
\operatorname{argmin}_{\left(w_{0}, v_{0}\right)} u^{T}\left(t^{i}, w_{0}, v_{0}\right) T^{T} T u\left(t^{i}, w_{0}, v_{0}\right) \\
\text { such that } \alpha_{1} w_{0}+\alpha_{2} v_{0}=x_{0}
\end{gathered}
$$

for a sequence $t^{i} \rightarrow \infty$. Note that the constraint $x_{0}$ fixed is now a linear variety in the new coordinates.

Lemma 4.1. $\alpha_{1}, \alpha_{2}$ are invertible if $\sigma(A) \subset C^{-}$and $R \geq 0$.

Proof. Note that $\left(\begin{array}{l}\alpha_{1} \\ \alpha_{3}\end{array}\right)$ spans the $n$-dimensional stable eigenspace and $\left(\begin{array}{l}\alpha_{2} \\ \alpha_{4}\end{array}\right)$ spans the $n$-dimensional unstable subspace. Standard results from Algebraic Riccati Equation theory [2] show that the stable and unstable subspaces of $H$ have the form

$$
\Upsilon=\operatorname{Im}\left(\begin{array}{l}
I \\
P
\end{array}\right)
$$

where $P$ is a solution of the Algebraic Riccati Equation

$$
A^{T} P+P A+\frac{4}{k} P R P+Q=0
$$

i.e., there exists an $\omega \in \mathbb{R}^{n \times n}$ such that

$$
\begin{aligned}
& \alpha_{1}=\omega, \\
& \alpha_{3}=P \omega,
\end{aligned}
$$

and thus $\alpha_{3}=P \alpha_{1}$. So if $x \in \operatorname{ker}\left(\alpha_{1}\right)$, then $x \in \operatorname{ker}\left(\alpha_{3}\right)$. However, since the combined vector $\left(\begin{array}{c}\alpha_{1} \\ \alpha_{3}\end{array}\right)^{T}$ must span $n$-dimensions, this is only possible if $x=0$. Therefore, $\operatorname{ker}\left(\alpha_{1}\right)=\{0\}$, and $\alpha_{1}$ is invertible. By the same method, $\alpha_{2}$ is nonsingular as well.

The constraint (4.4) can now be written as

$$
w_{0}=\alpha_{1}^{-1}\left(x_{0}-\alpha_{2} v_{0}\right) .
$$

Define

$$
\hat{u}\left(t, v_{0}\right)=\left(\begin{array}{c}
\hat{w}\left(t, v_{0}\right) \\
\hat{v}\left(t, v_{0}\right)
\end{array}\right)=\left(\begin{array}{l}
w\left(t, w_{0}\left(v_{0}\right), v_{0}\right) \\
v\left(t, w_{0}\left(v_{0}\right), v_{0}\right)
\end{array}\right)
$$


From Lemma 3.2, it is known that as $i \rightarrow \infty, v_{0}^{i} \rightarrow v_{0}^{*}$, where $\left(\begin{array}{c}w_{0}^{*} \\ v_{0}^{*}\end{array}\right)=u_{0}^{*}$ is on the stable manifold.

We consider $t^{i}$ in a compact interval $[0, \bar{t}], \bar{t}>0$. The rate of convergence is found using the mean value theorem. For any element $v_{0}^{i}$,

$$
\hat{v}\left(t, v_{0}^{i}\right)-\hat{v}\left(t, v_{0}^{*}\right)=\frac{\partial \hat{v}}{\partial v_{0}}\left(\hat{u}\left(t, \tilde{v}_{0}^{i}\right)\right)\left(v_{0}^{i}-v_{0}^{*}\right)
$$

for all $t \in\left[0, t^{i}\right]$, where $\tilde{v}_{0}^{i}$ lies on the line segment connecting $v_{0}^{i}$ and $v_{0}^{*}$.

It will be shown below that ${\frac{\partial \hat{v}}{\partial v_{0}}}^{-1}$ exists. Assuming for now that it does, rearrange (4.7) and take the norm of both sides to obtain

$$
\left\|v_{0}^{i}-v_{0}^{*}\right\| \leq \| \frac{\partial \hat{v}^{-1}}{\partial v_{0}}\left(\hat{u}\left(t, \tilde{v}_{0}^{i}\right)\|\| \hat{v}\left(t, v_{0}^{i}\right)-\hat{v}\left(t, v_{0}^{*}\right) \| .\right.
$$

Since $\left\|v_{0}^{i}-v_{0}^{*}\right\| \rightarrow 0$ as $i \rightarrow \infty,\left\|\hat{v}\left(t, v_{0}^{i}\right)-\hat{v}\left(t, v_{0}^{*}\right)\right\| \rightarrow 0$ for all $t$ in $\left[0, t^{i}\right]$. Therefore, if $\left\|\frac{\partial \hat{v}^{-1}}{\partial v_{0}}\right\|$ goes to zero, then $v_{0}^{i}$ must approach $v_{0}^{*}$ at least at the same rate.

We now calculate $\left\|\frac{\partial \hat{v}}{\partial v_{0}}{ }^{-1}\right\|$. First, use the chain rule on $\hat{u}$, i.e.,

$$
\begin{array}{r}
\frac{\partial \hat{u}}{\partial v_{0}}=\left(\begin{array}{c}
\frac{\partial \hat{w}}{\partial v_{0}} \\
\frac{\partial \hat{v}}{\partial v_{0}}
\end{array}\right)=\frac{\partial u}{\partial u_{0}}\left(\hat{u}\left(t, \tilde{v}_{0}^{i}\right)\right) \frac{\partial u_{0}}{\partial v_{0}} \\
=\left(\begin{array}{cc}
\frac{\partial w}{\partial w_{0}} & \frac{\partial w}{\partial v_{0}} \\
\frac{\partial v}{\partial w_{0}} & \frac{\partial v}{\partial v_{0}}
\end{array}\right)\left(\begin{array}{c}
-\alpha_{1}^{-1} \alpha_{2} \\
I
\end{array}\right),
\end{array}
$$

and therefore

$$
\frac{\partial \hat{v}}{\partial v_{0}}=\left.\left(\frac{\partial v}{\partial w_{0}}\left(-\alpha_{1}^{-1} \alpha_{2}\right)+\frac{\partial v}{\partial v_{0}}\right)\right|_{\hat{u}\left(t, \tilde{v}_{0}^{i}\right)} .
$$

Let $Z^{i}$ be the variational matrix of (4.1) at the $i$ th iteration, i.e., $Z^{i} \triangleq \frac{\partial u}{\partial u_{0}}\left(\hat{u}\left(t, \tilde{v}_{0}^{i}\right)\right)$. Then $Z^{i}$ satisfies the equation

$$
\begin{aligned}
\dot{Z}^{i} & =\left(A+B^{i}(t)\right) Z^{i}, \\
Z^{i}(0) & =I,
\end{aligned}
$$

where

$$
\left.A \triangleq\left(\begin{array}{cc}
\Lambda & 0 \\
0 & -\Lambda
\end{array}\right) \quad B^{i}(t) \triangleq\left(\begin{array}{ll}
\frac{\partial g_{1}}{\partial w} & \frac{\partial g_{1}}{\partial v} \\
\frac{\partial g_{2}}{\partial w} & \frac{\partial g_{2}}{\partial v}
\end{array}\right)\right|_{\hat{u}\left(t, \tilde{v}_{0}^{i}\right)} .
$$

Remark 4.1. Note that if the Hamiltonian is linear, then $B^{i}(t)=0$ and (4.10) can be solved directly. In that case, $\frac{\partial \hat{v}}{\partial v_{0}}=e^{-\Lambda t} I$, and $\left\|v_{0}^{i}-v_{0}^{*}\right\|=0\left(e^{\operatorname{Re} \lambda_{1} t}\right)$. Since $x_{0}$ is chosen near the origin, the nonlinear terms should not have a large effect on the variational system. See Figure 4.1 The following analysis seeks to measure the effect of this "perturbation" on the rate of convergence.

The following well-known theorem will be useful and is quoted here in its entirety. 
Theorem 4.1 ([11]). Let $f_{n}(t, x)$ be a sequence of functions which are defined and continuous in an open set $D$ and suppose that $\lim _{n \rightarrow \infty} f_{n}=f$ exists uniformly on any compact subset of D. Let $\left(t_{n}, \xi_{n}\right)$ be a sequence of points converging to a point $(\bar{t}, \bar{\xi})$. Let $\varphi_{n}(t)$ be any solution of the differential equation $\dot{x}=f_{n}(t, x)$ passing through the point $\left(t_{n}, \xi_{n}\right)$. If the solution $\varphi(t)$ of the differential equation $\dot{x}=f(t, x)$ which passes through the point $(\bar{t}, \bar{\xi})$ is defined on the interval $[a, b]$ and is unique, then $\varphi_{n}(t)$ is defined on $[a, b]$ for all sufficiently large $n$ and $\varphi_{n}(t) \rightarrow \varphi(t)$ uniformly on this interval as $n \rightarrow \infty$.

Since $v_{0}^{i}$ approaches $v_{0}^{*}$, and $\tilde{v}_{0}^{i}$ is on the line joining $v_{0}^{i}$ to $v_{0}^{*}$, it follows that $\tilde{v}_{0}^{i}$ approaches $v_{0}^{*}$ as $i \rightarrow \infty$. Fix a time $t^{i}=\bar{t}$ from the sequence of times. From Theorem 4.1. it follows that $\hat{u}\left(t, \tilde{v}_{0}^{j}\right)$ approaches $\hat{u}\left(t, v_{0}^{*}\right)$ uniformly for $t \in[0, \bar{t}]$ as $j \rightarrow \infty$. Now define $B^{*}(t)=\frac{\partial g}{\partial u}\left(u\left(t, v_{0}^{*}\right)\right)$. Then

Lemma 4.2. Let $g(\cdot) \in C^{2}$. Then $B^{j}(t) \rightarrow B^{*}(t)$ uniformly on the compact interval $t \in[0, \bar{t}]$ for any finite $\bar{t}$.

Proof.

$$
\begin{aligned}
\lim _{j \rightarrow \infty} B^{j}(t) & =\lim _{j \rightarrow \infty} \frac{\partial g}{\partial u}\left(\hat{u}\left(t, \hat{v}_{0}^{j}\right)\right) \\
& =\frac{\partial g}{\partial u}\left(\lim _{j \rightarrow \infty}\left(\hat{u}\left(t, \hat{v}_{0}^{j}\right)\right)\right. \\
& =\frac{\partial g}{\partial u}\left(\hat{u}\left(t, \lim _{j \rightarrow \infty} \tilde{v}_{0}^{j}\right)\right) \\
& =\frac{\partial g}{\partial u}\left(\tilde{u}\left(t, v_{0}^{*}\right)\right) \triangleq B^{*}(t) .
\end{aligned}
$$

Now let $Z^{*}(t)$ be the solution to the variational system along the stable manifold, i.e., it solves the equation $\dot{Z}=\left(A+B^{*}(t)\right) Z, Z(0)=I$. Applying Theorem 4.1 again, it follows that

$$
Z^{j}(t) \rightarrow Z^{*}(t) \quad \text { as } j \rightarrow \infty \text { uniformly for } t \in[0, \bar{t}] .
$$

Trajectories lying on the stable manifold approach the origin exponentially [12]. Therefore, along the stable manifold, asymptotic results on the solutions of timevarying differential equations, can be applied. We quote one such result in Theorem 4.2 .

Theorem 4.2. [13] Consider a system $\dot{z}=(A+B(t)) z$. Suppose $A$ has simple characteristic roots, $\lambda_{k}, k=1, \ldots, n$ satisfying $\operatorname{Re} \lambda_{k-1} \leq \operatorname{Re} \lambda_{k}<\operatorname{Re} \lambda_{k+1} \leq$ $\operatorname{Re} \lambda_{k+2}$ and $\int^{\infty}\|B(t)\| d t<\infty$. Then there are $n$ solutions $z_{1}, z_{2}, \ldots, z_{n}$ such that

$$
z_{k}=e^{\lambda_{k} t}\left(e_{k}+o(1)\right)
$$

as $t \rightarrow \infty$, where $e_{k}$ is the $k$ th unit vector.

Since the trajectories along the stable manifold approach the origin exponentially, $\int^{\infty}\left\|B^{*}(t)\right\| d t$ converges, and Theorem 4.2 can be applied to the system

$$
\dot{w}=\left(A+B^{*}(t)\right) w .
$$


Combine the $n$ solutions which satisfy (4.12) into the matrix $W(t)$. However, since $W(0)$ is not the identity, $W(t)$ is not the variational matrix $Z^{*}(t)$. Using (4.12) it is easy to check that

$$
\begin{aligned}
& W(t)=e^{\left[\begin{array}{cc}
\wedge & 0 \\
0 & -\wedge
\end{array}\right] t}(I+\psi(t)), \\
& Z^{*}(t)=W(t) W(0)^{-1},
\end{aligned}
$$

where $\psi(t)=o(1)$ as $t \rightarrow \infty$.

The following theorem will be used to bound $\psi(0)$.

Theorem 4.3 ([13]). Again, consider a system $\dot{z}=(A+B(t)) z$, and suppose $A$ has simple eigenvalues $\lambda_{k}$, ordered as in Theorem 4.2. and $\|B(t)\| \rightarrow 0$ as $t \rightarrow \infty$. Then each solution $z_{1}, z_{2}, \ldots, z_{n}$ satisfies the inequality

$$
c_{2} e^{\operatorname{Re} \lambda_{k} t-d_{2} \int_{0}^{t}\|B\| d t} \leq\left\|z_{k}\right\| \leq c_{1} e^{\operatorname{Re} \lambda_{k} t+d_{1} \int_{0}^{t}\|B\| d t}
$$

for $t \geq 0$, with $c_{1}, c_{2}, d_{1}, d_{2}$ all positive constants. Furthermore, each solution $z_{k}$, $k=1, \ldots, n$, also satisfies the integral equation

$$
z_{k}(t)=e^{\lambda_{k} t} e_{k}+\int_{0}^{t} Y_{1}(t-s) B(s) z(s) d s-\int_{t}^{\infty} Y_{2}(t-s) B(s) z(s) d s,
$$

where

$$
Y_{1}(t) \triangleq \operatorname{diag}\left(e^{\lambda_{1} t}, \ldots, e^{\lambda_{k} t}, 0, \ldots, 0\right) \text { and } Y_{2}(t) \triangleq \operatorname{diag}\left(0, \ldots, 0, e^{\lambda_{k+1} t}, \ldots, e^{\lambda_{n} t}\right) \text {. }
$$

Theorem 4.3 can be applied to each column of $W$, since $\left\|B^{*}(t)\right\| \rightarrow 0$ as $t \rightarrow \infty$. Using (4.16) at $t=0$ on $w_{k}$,

$$
w_{k}(0)=e_{k}-\int_{0}^{\infty} Y_{2}\left(-t_{1}\right) B^{*}\left(t_{1}\right) z_{k}\left(t_{1}\right) d t_{1} .
$$

So for $w_{k}$, the $k$ th column of $W$, the $k$ th column of $\psi(0), \psi_{k}(0)$, is equal to the integral term of (4.17).

Lemma 4.3. Given $\varepsilon>0$. For all $x_{0}$ sufficiently close to $0,\left\|B^{*}(t)\right\|<\varepsilon$, for all $t>0$.

Proof. Solutions starting on the stable manifold remain there and approach zero exponentially [12]. For any $u_{0}^{*}=T^{-1}\left(\begin{array}{l}x_{0} \\ p_{0}^{*}\end{array}\right)$ on the stable manifold it follows that

$$
\left\|u\left(t, u_{0}^{*}\right)\right\| \rightarrow 0 \quad \text { as } t \rightarrow \infty \text {. }
$$

By choosing $\left\|x_{0}\right\|$ small so that $p_{0}^{*}$ is also small, $\left\|u_{0}^{*}\right\|$ is small and

$$
\left\|u\left(t, u_{0}^{*}\right)\right\|<\varepsilon_{1} \quad \text { fort }>0 .
$$

From (4.2) letting $\varepsilon_{1}=\varepsilon / K$

$$
\left\|B^{*}(t)\right\|=\| \frac{\partial g}{\partial v}\left(\tilde{u}\left(t, v_{0}^{*}\right)\|\leq K\| \tilde{u}\left(t, v_{0}^{*}\right) \| \leq K \varepsilon_{1}=\varepsilon .\right.
$$

Lemma 4.4. Given $\varepsilon>0$. By choosing $x_{0}$ sufficiently close to 0 ,

$$
\int_{0}^{\infty}\left\|B^{*}(t)\right\| d t<\varepsilon
$$




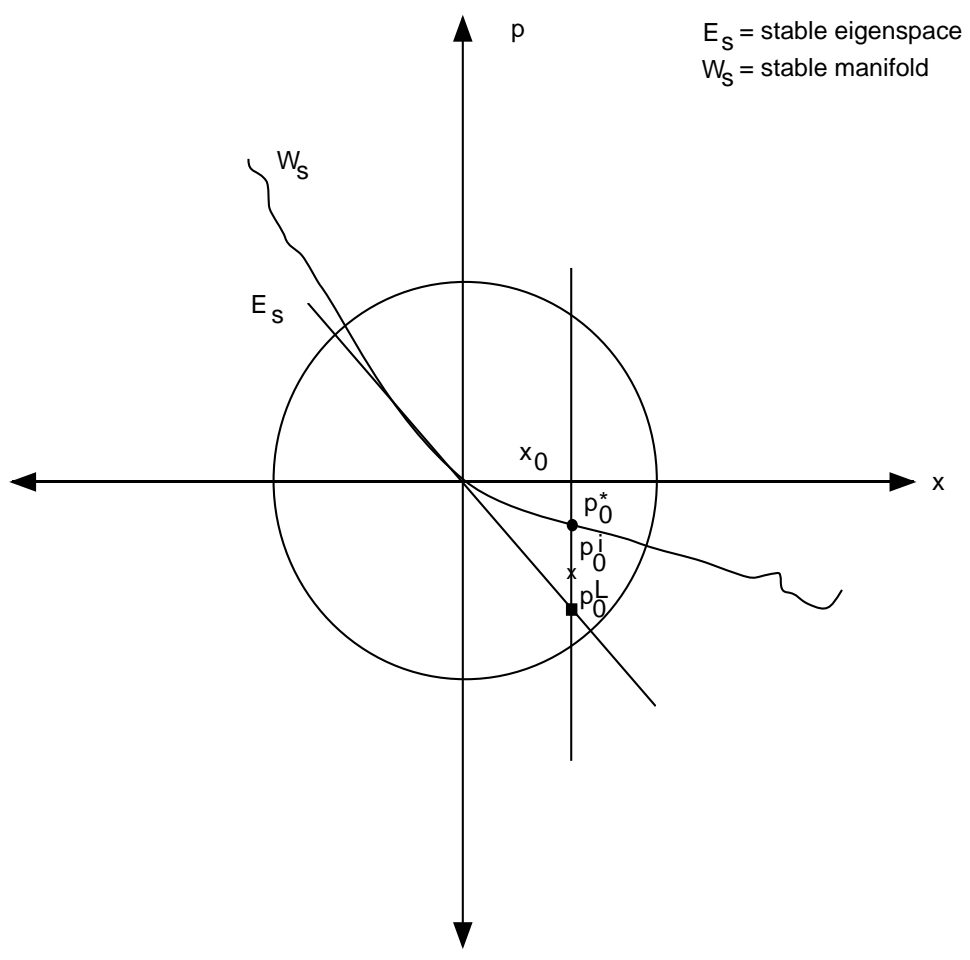

FiguRE 4.1. Stable subspace and manifold for Hamiltonian system

Proof. Since $\int^{\infty}\left\|B^{*}(t)\right\| d t<\infty$, there is a $T>0$ such that $\int_{T}^{\infty}\left\|B^{*}(t)\right\| d t<\frac{\varepsilon}{2}$. Using Lemma 4.3, choose $x_{0}$ so that $\left\|B^{*}(t)\right\|<\frac{\varepsilon}{2 T}$. Then

$$
\begin{aligned}
\int_{0}^{\infty}\left\|B^{*}(t)\right\| d t & =\int_{0}^{T}\left\|B^{*}(t)\right\| d t+\int_{T}^{\infty}\left\|B^{*}(t)\right\| d t \\
& \leq \frac{\varepsilon}{2 T} T+\frac{\varepsilon}{2}=\varepsilon .
\end{aligned}
$$

Now by choosing $\left\|x_{0}\right\|$ sufficiently small, (4.15) becomes

$$
\left\|z_{k}\right\| \leq c_{1} e^{\operatorname{Re} \lambda_{k} t+d_{1} \int_{0}^{\infty}\left\|B^{*}\left(t_{1}\right)\right\| d t_{1}} \leq c_{1} e^{d_{1} \varepsilon} e^{\operatorname{Re} \lambda_{k} t}
$$

and the integral term in (4.17) is bounded by

$$
\begin{aligned}
\left\|\int_{0}^{\infty} Y_{2}\left(-t_{1}\right) B\left(t_{1}\right) z_{k}\left(t_{1}\right) d t_{1}\right\| & \leq c_{1} e^{d_{1} \varepsilon} \int_{0}^{\infty} e^{\operatorname{Re}\left(\lambda_{k}-\lambda_{k+1}\right) t_{1}}\left\|B\left(t_{1}\right)\right\| d t_{1} \\
& \leq c_{1} e^{d_{1} \varepsilon} \varepsilon
\end{aligned}
$$

since $\operatorname{Re}\left(\lambda_{k}-\lambda_{k+1}\right) \leq 0$. Therefore $\|\psi(0)\|$ can be made arbitrarily small, by choosing $\left\|x_{0}\right\|$ sufficiently small. That is

$$
\|\psi(0)\|=o(1) \quad \text { as }\left\|x_{0}\right\| \rightarrow 0 .
$$

Define $z_{k}^{*}(t)$ as the $k$ th column of $Z^{*}(t)$. Using the above result on $W(0), z_{k}^{*}(t)=$ $e^{\lambda_{k} t}\left(\delta_{i k}+o(1)\right)$ as $\left\|x_{0}\right\| \rightarrow 0$. Similarly, define $z_{k}^{i}(t)$ as the $k$ th column of $Z^{i}(t)$. 
Introduce the notation $o_{i}(1)$ to mean $o(1)$ as $i \rightarrow \infty, o_{t}(1)$ to mean $o(1)$ as $t \rightarrow \infty$, and $o_{x_{0}}(1)$ to mean $o(1)$ as $\left\|x_{0}\right\| \rightarrow 0$. Using this notation, for fixed $t^{i}$, it follows from (4.10) with $\bar{t}=t^{i}$ that

$$
\left\|z^{i}\left(t^{i}\right)-z^{*}\left(t^{i}\right)\right\|=o_{i}(1)
$$

from (4.14) that

$$
z^{*}\left(t^{i}\right)=e^{\left[\begin{array}{cc}
\wedge & 0 \\
0 & -\wedge
\end{array}\right] t^{i}}\left(I+\psi\left(t^{i}\right)\right)(I+\psi(0))^{-1},
$$

where

$$
\left\|\psi\left(t^{i}\right)\right\|=o_{t^{i}}(1)
$$

and from (4.19) that

$$
\|\psi(0)\|=o_{x_{0}}(1)
$$

\section{Theorem 4.4 .}

$$
\left\|p_{0}^{i}-p_{0}^{*}\right\| \approx 0\left(e^{\operatorname{Re} \lambda_{1} t^{i}}\right)+o_{i}(1)+o_{x_{0}}(1)
$$

Proof. In the following calculations we retain only terms which are first order in $o(1)$. Using (4.20a), 4.20b), (4.20c), and 4.20d), we have

$$
\begin{aligned}
\frac{\partial u}{\partial u_{0}}\left(\hat{u}\left(t^{i}, \tilde{v}_{0}^{i}\right)\right) & \equiv Z^{i}\left(t^{i}\right) \\
& =Z^{*}\left(t^{i}\right)+o_{i}(1) \\
& =e^{\left[\begin{array}{cc}
\wedge & 0 \\
0 & -\wedge
\end{array}\right] t^{i}}\left(I+\psi\left(t^{i}\right)\right)(I+\psi(0))^{-1}+o_{i}(1) \\
& =e^{\left[\begin{array}{cc}
\wedge & 0 \\
0 & -\wedge
\end{array}\right] t^{i}\left(I+\psi\left(t^{i}\right)-\psi(0)+\psi\left(t^{i}\right) \psi(0)\right)+o_{i}(1)} \\
& =e^{\left[\begin{array}{cc}
\wedge & 0 \\
0 & -\wedge
\end{array}\right] t^{i}\left(I-\psi(0)+o_{t^{i}}(1)\right)+o_{i}(1) .}
\end{aligned}
$$

Let

$$
\psi(t)=\left[\begin{array}{ll}
\psi_{11}(t) & \psi_{12}(t) \\
\psi_{21}(t) & \psi_{22}(t)
\end{array}\right]
$$

Then

$$
\begin{aligned}
\frac{\partial v}{\partial w_{0}}\left(\hat{u}\left(t^{i}, \tilde{v}_{0}^{j}\right)\right) & =e^{-\wedge t^{i}}\left(-\psi_{21}(0)+o_{t^{i}}(1)\right)+o_{i}(1), \\
\frac{\partial v}{\partial v_{0}}\left(\hat{u}\left(t^{i}, \tilde{v}_{0}^{j}\right)\right) & =e^{-\wedge t^{i}}\left(I-\psi_{22}(0)+o_{t^{i}}^{(1)}\right)+o_{i}(1) .
\end{aligned}
$$


Substituting (4.22) in (4.9) gives

$$
\begin{aligned}
\frac{\partial \hat{v}}{\partial v_{0}}\left(\hat{u}\left(t^{i}, \hat{v}_{0}^{i}\right)\right)= & {\left[e^{-\wedge t^{i}}\left(-\psi_{21}(0)+o_{t^{i}}(1)\right)+o_{i}(1)\right]\left(-\alpha_{1}^{-1} \alpha_{2}\right) } \\
& +\left[e^{-\wedge t^{i}}\left(I-\psi_{22}(0)+o_{t^{i}}(1)\right)+o_{i}(1)\right] \\
= & {\left[e^{-\wedge t^{i}}\left(o_{x_{0}}(1)+o_{t^{i}}(1)\right)+o_{i}(1)\right]\left(-\alpha_{1}^{-1} \alpha_{2}\right) } \\
& +\left[e^{-\wedge t^{i}}\left(I+o_{x_{0}}(1)+o_{t^{i}}(1)\right)+o_{i}(1)\right] \\
= & e^{-\wedge t^{i}}\left(I+o_{x_{0}}(1)+o_{t^{i}}(1)\right)+o_{i}(1)
\end{aligned}
$$

Therefore

$$
\begin{aligned}
\left(\frac{\partial \hat{v}}{\partial v_{0}}\right)^{-1}\left(\hat{u}\left(t^{i}, \tilde{v}_{0}^{i}\right)\right) & =\left[e^{-\wedge t^{i}}\left(I+o_{x_{0}}(1)+o_{t^{i}}(1)\right)+o_{i}(1)\right]^{-1} \\
& =\left[e^{-\wedge t^{i}}\left(\left(I+o_{x_{0}}(1)+o_{t^{i}}(1)\right)+e^{\wedge t^{i}} o_{i}(1)\right)\right]^{-1} \\
& =\left[I+o_{x_{o}}(1)+o_{t^{i}}(1)+e^{\wedge t^{i}} o_{i}(1)\right]^{-1} e^{\wedge t^{i}} \\
& =\left[I+o_{x_{o}}(1)+o_{t^{i}}(1)+e^{\wedge t^{i}} o_{i}(1)\right] e^{\wedge t^{i}}
\end{aligned}
$$

and

$$
\|\left(\frac{\partial \tilde{v}}{\partial v_{0}}\right)^{-1}\left(\hat{u}\left(t^{i}, \tilde{v}_{0}^{i}\right) \|=\left(1+o_{x_{o}}(1)+o_{t^{i}}(1)+o_{i}(1)\right) e^{\left(\operatorname{Re} \lambda_{1}\right) t^{i}} .\right.
$$

Now from (4.8) with $t=t^{i}$, it follows that

$$
\left\|v_{0}^{i}-v_{0}^{*}\right\|=0\left(e^{\operatorname{Re} \lambda_{1} t^{i}}\right) \quad \text { as } i \rightarrow \infty, t^{i} \rightarrow \infty, x_{0} \rightarrow 0
$$

From (4.5)

$$
\begin{aligned}
& w_{0}^{i}=\alpha_{1}^{-1}\left(x_{0}-\alpha_{2} v_{0}^{i}\right), \quad w_{0}^{*}=\alpha_{1}^{-1}\left(x_{0}-\alpha_{2} v_{0}^{*}\right) \\
& w_{0}^{i}-w_{0}^{*}=-\alpha_{2}\left(v_{0}^{i}-v_{0}^{*}\right) .
\end{aligned}
$$

Therefore

$$
\begin{aligned}
\left\|p_{0}^{i}-p_{0}^{*}\right\|=\left\|z_{0}^{i}-z_{0}^{*}\right\| & \\
=\left\|T\left(u_{0}^{i}-u_{0}^{*}\right)\right\| & \leq C\left\|u_{0}^{i}-u_{0}^{*}\right\|=0\left(e^{\operatorname{Re} \lambda_{1} t^{i}}\right) \\
& \text { as } i \rightarrow \infty, \quad t^{i} \rightarrow \infty, \quad x_{o} \rightarrow 0 .
\end{aligned}
$$

The following example, although not a Hamiltonian system, illustrates the ideas in the proof of the previous theorem.

\section{Example 4.1.}

$$
\begin{aligned}
& \dot{w}=-w, \quad u=\left[\begin{array}{l}
w \\
v
\end{array}\right], \\
& \dot{v}=v+\varepsilon w^{2}
\end{aligned}
$$

subject to

$$
\alpha_{1} w(0)+\alpha_{2} v(0)=x_{0} .
$$

It is easily verified that the general solution to (4.25) is

$$
\begin{aligned}
& w(t)=w(0) e^{-t} \\
& v(t)=\left(\varepsilon \frac{w^{2}(0)}{3}+v(0)\right) e^{t}-\varepsilon \frac{w^{2}(0)}{3} e^{-2 t} .
\end{aligned}
$$


The stable manifold of (4.25) is

$$
\varepsilon \frac{w^{* 2}(0)}{3}+v^{*}(0)=0
$$

The variational equation corresponding to (4.25) is

$$
\begin{aligned}
\left(\frac{\partial \dot{u}}{\partial u_{0}}\right) & =\left[\begin{array}{cc}
-1 & 0 \\
2 \varepsilon w(t) & 1
\end{array}\right]\left(\frac{\partial u}{\partial_{0}}\right) \\
& =\left(\left[\begin{array}{cc}
-1 & 0 \\
0 & 1
\end{array}\right]+B(t)\right)\left(\frac{\partial u}{\partial u_{0}}\right),
\end{aligned}
$$

where

$$
B(t)=\left[\begin{array}{cc}
0 & 0 \\
2 \varepsilon w(t) & 0
\end{array}\right]=\left[\begin{array}{cc}
0 & 0 \\
2 \varepsilon w(0) e^{-t} & 0
\end{array}\right] .
$$

Clearly $\int_{0}^{\infty}\|B(t)\| d t<\infty$, and $\|B(t)\|$ can be made arbitrarily small for all $t>0$ by choosing $w(0)$ small.

The transition matrix $Z(t)$ for (4.28) is

$$
\begin{aligned}
& Z(t)=Z(t, w(0))=\left[\begin{array}{cc}
e^{-t} & 0 \\
\frac{2 \varepsilon w(0)}{3}\left(e^{t}-e^{-2 t}\right) & e^{t}
\end{array}\right]
\end{aligned}
$$

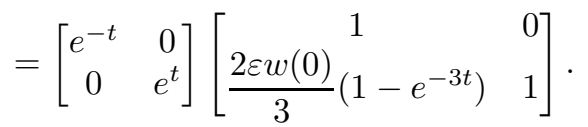

The 2 solutions $z_{1}(t), z_{2}(t)$ in 4.12 can be computed by writing $z_{i}(t)=Z(t) c_{i}$, $i=1,2$, and substituting in 4.16) to determine $c_{i}$.

This gives

$$
z_{1}(t)=\left[\begin{array}{c}
e^{-t} \\
-\frac{2 \varepsilon}{3} w(0) e^{-2 t}
\end{array}\right], \quad z_{2}(t)=\left[\begin{array}{c}
0 \\
e^{t}
\end{array}\right]
$$

so

$$
\begin{aligned}
W(t)=W(t, w(0)) & =\left[z_{1}(t) \vdots z_{2}(t)\right] \\
& =\left[\begin{array}{cc}
e^{-t} & 0 \\
0 & e^{t}
\end{array}\right](I+\psi(t)),
\end{aligned}
$$

where

$$
\begin{aligned}
\psi(t) & =\left[\begin{array}{cc}
0 & 0 \\
-\frac{2 \varepsilon}{3} w(0) e^{-3 t} & 0
\end{array}\right], \\
W(t) W^{-1}(0) & \left.=\left[\begin{array}{cc}
e^{-t} & 0 \\
-\frac{2 \varepsilon}{3} w(0) e^{-2 t} & e^{t}
\end{array}\right]\left[\begin{array}{cc}
1 & 0 \\
\frac{2 \varepsilon}{3} w(0) & 1
\end{array}\right]\right]^{-1} \\
& =\left[\begin{array}{cc}
e^{-t} & 0 \\
0 & e^{t}
\end{array}\right]\left[\begin{array}{cc}
\frac{2 \varepsilon}{3} w(0)\left(1-e^{-3 t}\right) & 1
\end{array}\right]=Z(t) \\
& =\left[\begin{array}{cc}
e^{-t} & 0 \\
0 & e^{t}
\end{array}\right](I+\psi(t)-\psi(0)) .
\end{aligned}
$$

Note that $\psi(t) \rightarrow 0$ as $t \rightarrow \infty$, and for $w(0)$ sufficiently small, $\psi(0)$ is small. Also, for each fixed $t, z\left(t, w_{i}(0)\right) \rightarrow z\left(t, w^{*}(0)\right)$ as $w_{i}(0) \rightarrow w^{*}(0)$. 
Equation (4.24) shows that $\left\|w_{i}(0)-w^{*}(0)\right\|=0\left(e^{-t^{i}}\right)$ as $i \rightarrow \infty$. This bound is not sharp. A calculation for small $\varepsilon$ shows that, in fact, $\left\|w_{i}(0)-w^{*}(0)\right\|=0\left(e^{-4 t^{i}}\right)$ as $i \rightarrow \infty$. This is the rate of convergence of the linearized system of (4.25) as shown in the next section.

4.2. Linear systems. It is now not assumed that the Hamiltonian system has distinct eigenvalues.

The main result in this section is

$$
\left\|p_{0}^{i}-p_{0}^{*}\right\|=0\left(e^{2 \operatorname{Re} \lambda_{1} t^{i}}\right) .
$$

If the stable and unstable eigenspaces are orthogonal, then

$$
\left\|p_{0}^{i}-p_{0}^{*}\right\|=0\left(e^{4 \operatorname{Re} \lambda_{1} t^{i}}\right)
$$

Define $T$ as the set of generalized eigenvectors of $\bar{H}$ such that $D \triangleq T^{-1} \bar{H} T$ is in Jordan block form. Furthermore, partition $T$ into the four $n \times n$ submatrices

$$
T=\left(\begin{array}{ll}
\alpha_{1} & \alpha_{2} \\
\alpha_{3} & \alpha_{4}
\end{array}\right)
$$

Again, to facilitate the analysis, the system is put into "eigenvector" form by making the coordinate transformation $T u=z$, where $u^{T} \triangleq\left(w^{T} v^{T}\right)$. In the new coordinates the Hamiltonian system (2.6) with $h(z) \equiv 0$ is $\dot{u}=D u$, or more explicitly

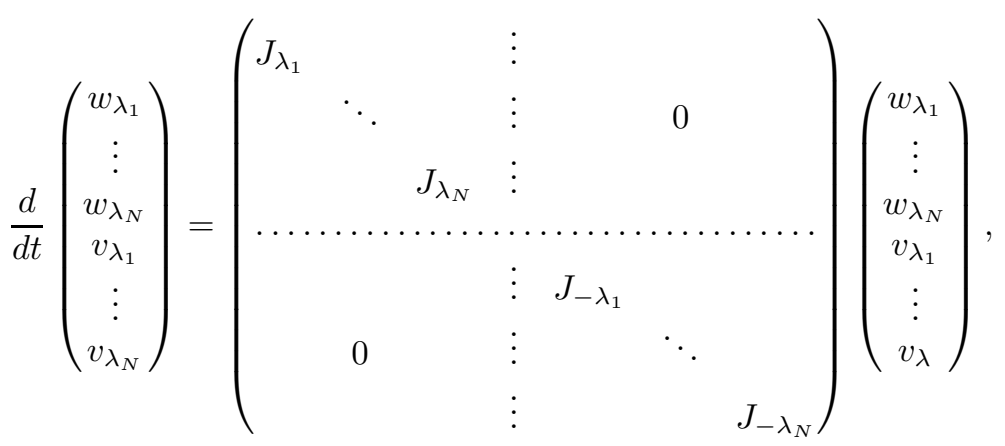

where $J_{\lambda_{i}}$ is the Jordan block corresponding to the eigenvalue $\lambda_{i}$ of $\bar{H}$, and $w_{\lambda_{i}}$, $v_{\lambda_{i}} \in \mathbb{R}^{k_{i}}$, where $k_{i}$ is the multiplicity of $\lambda_{i} . T$ is constructed such that

$$
\operatorname{Re}\left(\lambda_{n}\right) \leq \operatorname{Re}\left(\lambda_{n-1}\right) \leq \cdots \leq \operatorname{Re}\left(\lambda_{1}\right)<0 .
$$

Recall that the algorithm solves the minimization problem

$$
\begin{gathered}
\min _{p_{0}} F\left(t, x_{0}, p_{0}\right)=z^{T}\left(t, x_{0}, p_{0}\right) z\left(t, x_{0}, p_{0}\right) \\
\text { such that }\left(\begin{array}{ll}
I & 0
\end{array}\right) z_{0}=x_{0}
\end{gathered}
$$

for a fixed time $t$. Using the transformation $T u=z$, the problem in the new coordinates is

$$
\begin{gathered}
\min _{u_{0}} F\left(t, u_{0}\right)=u_{0} e^{D^{T} t} X e^{D t} u_{0} \\
\text { such that }(I \quad 0) T u_{0}=x_{0}
\end{gathered}
$$

where $X \triangleq T^{T} T$. 
Because the time $t$ is fixed, (4.34) can be solved analytically using Lagrangian multipliers. Define the costate vector $\mu \triangleq\left[\mu_{1} \cdots \mu_{n}\right]$ and the objective function

$$
J \triangleq F+\mu\left(\alpha_{1} w_{0}+\alpha_{2} v_{0}-x_{0}\right) .
$$

Then for any finite $t$, the minimizing initial condition $u_{0}(t)$ of (4.34) also satisfies the $3 n$-dimensional linear system

$$
\begin{gathered}
\frac{\partial J}{\partial u_{0}}=2 e^{D^{T} t} X e^{D t} u_{0}+T^{T}\left(\begin{array}{ll}
I & 0
\end{array}\right)^{T} \mu^{T}=0, \\
\frac{\partial J}{\partial \mu}=\left(\begin{array}{ll}
I & 0
\end{array}\right) T u_{0}=x_{0} .
\end{gathered}
$$

Solving equation (4.37) gives the expression

$$
u_{0}(t)=-\frac{1}{2}\left(e^{D^{T} t} X e^{D t}\right)^{-1} T^{T}\left(\begin{array}{l}
I \\
0
\end{array}\right) \mu^{T},
$$

which can be substituted into (4.38) to obtain

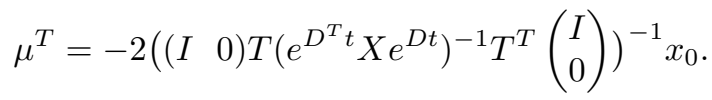

Combining (4.39) and (4.40), the vector $u_{0}(t)$ found by the algorithm is, for any fixed time $t$,

$$
\begin{aligned}
u_{0}(t)=\left(\begin{array}{c}
w_{0}(t) \\
v_{0}(t)
\end{array}\right) & =\left(e^{D^{T} t} X e^{D t}\right)^{-1} T^{T}\left(\begin{array}{l}
I \\
0
\end{array}\right)\left(\left(\begin{array}{ll}
I & 0
\end{array}\right) T\left(e^{D^{T} t} X e^{D t}\right)^{-1} T^{T}\left(\begin{array}{l}
I \\
0
\end{array}\right)\right)^{-1} x_{0} \\
& \triangleq\left(e^{D^{T} t} X e^{D t}\right)^{-1}\left(\begin{array}{c}
\alpha_{1}^{T} \\
\alpha_{2}^{T}
\end{array}\right) G^{-1}(t) x_{0} .
\end{aligned}
$$

Although Lemma 3.2 states that for nonlinear systems $u_{0}(t) \rightarrow u_{0}^{*}$, as $t \rightarrow \infty$, for linear systems this can be shown directly. Toward that end, define the following partitions

$$
X^{-1} \triangleq\left(\begin{array}{cc}
X_{1} & X_{2} \\
X_{2}^{T} & X_{3}
\end{array}\right), \quad e^{D t} \triangleq\left(\begin{array}{cc}
e^{\Lambda t} & 0 \\
0 & e^{-\Lambda t}
\end{array}\right) .
$$

From the definition of $D, e^{\Lambda t} \rightarrow 0$ as $t$ increases and $e^{-\Lambda t}$ grows exponentially in $t$.

From (4.41) it follows that for $v_{0}(t)$

$$
v_{0}(t)=\left(e^{\Lambda t} X_{2}^{T} e^{-\Lambda^{T} t} \alpha_{1}^{T}+e^{\Lambda t} X_{3} e^{\Lambda^{T} t} \alpha_{2}^{T}\right) G^{-1}(t) x_{0} .
$$

It will be shown that as $t$ increases, $v_{0}(t)$ approaches zero exponentially.

Expand $G(t)$ using the partitions of $D, T$ and $X$ to obtain

$$
\begin{aligned}
G(t)=( & \alpha_{1} e^{-\Lambda t} X_{1} e^{-\Lambda^{T} t} \alpha_{1}^{T}+\alpha_{1} e^{-\Lambda t} X_{2} e^{\Lambda^{T} t} \alpha_{2}^{T} \\
& \left.+\alpha_{2} e^{\Lambda t} X_{2}^{T} e^{-\Lambda^{T} t} \alpha_{1}^{T}+\alpha_{2} e^{\Lambda t} X_{3} e^{\Lambda^{T} t} \alpha_{2}^{T}\right) .
\end{aligned}
$$

Factoring the first term on the left and right gives

$$
\begin{aligned}
G(t)=\alpha_{1} e^{-\Lambda t}\left(X_{1}+e^{\Lambda t} \alpha_{1}^{-1}\left\{\alpha_{1} e^{-\Lambda^{T} t}\right.\right. & X_{2} e^{\Lambda^{T} t} \alpha_{2}^{T}+\alpha_{2} e^{\Lambda t} X_{2}^{T} e^{-\Lambda^{T} t} \alpha_{1}^{T} \\
& \left.\left.+\alpha_{2} e^{\Lambda^{T} t} X_{3} e^{\Lambda^{T} t} \alpha_{2}^{T}\right\} \alpha_{1}^{-T} e^{\Lambda^{T} t}\right) e^{-\Lambda^{T} t} \alpha_{1}^{T},
\end{aligned}
$$


which is equivalent to

$$
\begin{aligned}
G(t)=\alpha_{1} e^{-\Lambda t}\left(X_{1}\right. & +X_{2} e^{\Lambda^{T} t} \alpha_{2}^{T} \alpha_{1}^{-T} e^{\Lambda^{T} t} \\
& \left.+e^{\Lambda t} \alpha_{1}^{-1} \alpha_{2} e^{\Lambda t}\left(X_{2}^{T}+X_{3} e^{\Lambda^{T} t} \alpha_{2}^{T} \alpha_{1}^{-T} e^{\Lambda^{T} t}\right)\right) e^{-\Lambda^{T} t} \alpha_{1}^{T} .
\end{aligned}
$$

Note that the middle term has a factor $e^{\Lambda t}$, and therefore approaches $X_{1}$ as $t$ increases. Thus, for $t$ large enough,

$$
G^{-1}(t) \rightarrow \alpha_{1}^{-T} e^{\Lambda^{T} t} X_{1}^{-1} e^{\Lambda t} \alpha_{1}^{-1} .
$$

Substituting this approximation into (4.43) gives the large time approximation for $v_{0}$ :

$$
\begin{aligned}
v_{0}(t) & \rightarrow\left(e^{\Lambda t} X_{2}^{T} e^{-\Lambda^{T} t} \alpha_{1}^{T}+e^{\Lambda t} X_{3} e^{\Lambda^{T} t} \alpha_{2}^{T}\right) \alpha_{1}^{-T} e^{\Lambda^{T} t} X_{1}^{-1} e^{\Lambda t} \alpha_{1}^{-1} x_{0} \\
& \rightarrow\left(e^{\Lambda t} X_{2}^{T} X_{1}^{-1} e^{\Lambda t} \alpha_{1}^{-1}+e^{\Lambda^{T} t} X_{3} e^{\Lambda^{T} t} \alpha_{2}^{T} G^{-1}(t)\right) x_{0} .
\end{aligned}
$$

Again, because $\Lambda$ contains only negative eigenvalues of $\bar{H}$, (4.48) will approach zero as time increases.

The rate of convergence is determined by considering the "slowest" exponential function of (4.48), which will depend on the structure of $\sigma(\bar{H})$. For example, suppose $\bar{H}$ has distinct eigenvalues. By combining the terms of (4.48) into one expression, it is seen that each element of $v_{0}(t)$ will contain a linear combination of the functions $e^{\left(\lambda_{i}+\lambda_{j}\right) t}$ for $i, j=1 \cdots n$. Therefore, the algorithm will converge to the stable manifold exponentially at a rate of $2 \operatorname{Re} \lambda_{1}$.

Now suppose $\lambda_{1}$ has multiplicity $k_{1}>1$. In this case, the corresponding basis solution of the Hamiltonian system grows like $t^{k_{1}-1} e^{\lambda_{1} t}$. Hence, for repeated eigenvalues, the rate of convergence will be slowed by a factor of $t^{2 k_{1}-2}$.

From (4.48), it is clear that if the first term equals zero, the rate of convergence is doubled to an exponential rate of $4 \operatorname{Re} \lambda_{1}$. This can occur, for example, when $X_{2}=0$. Because $X=\left(T^{T} T\right)^{-1}$ and $T$ is the set of eigenvectors of $\bar{H}, X_{2}=0$ occurs when the stable and unstable subspaces are orthogonal to one another.

Of course orthogonality of the stable and unstable spaces is a specific case, but the higher rate of convergence can be achieved for any system by reformulating the problem. For example, instead of minimizing $F=\left\|z\left(t, z_{0}\right)\right\|^{2}$, diagonalize the Hamiltonian first and run the algorithm on the function $\tilde{F}=\left\|u\left(t, u_{0}\right)\right\|^{2}$ instead. The new sequence of initial conditions, $\tilde{u}_{0}^{i}(t)$ will satisfy (4.41) as well, except $X^{-1}$ is now replaced by the identity, or more specifically, $X_{1}=X_{3}=I$, and $X_{2}=0$. This can be seen explicitly in the following example.

Example 4.2. Consider a two-dimensional linear diagonalized Hamiltonian system

$$
\begin{aligned}
\dot{w} & =\lambda w, \quad \lambda<0, \\
\dot{v} & =-\lambda v .
\end{aligned}
$$

The stable manifold is the subspace $v=0$.

The algorithm minimizes the function

$$
F\left(t, w_{0}, v_{0}\right)=\left(\begin{array}{ll}
w & v
\end{array}\right)\left(\begin{array}{cc}
x_{1} & \frac{x_{2}}{2} \\
\frac{x_{2}}{2} & x_{3}
\end{array}\right)\left(\begin{array}{c}
w \\
v
\end{array}\right),
$$

where $x=T^{T} T$, over $\left(w_{0}, v_{0}\right)$ subject to the constraint

$$
a w_{0}+b v_{0}=c .
$$


The optimal initial conditions can be expressed as a function of time to be

$$
\begin{aligned}
& w_{0}(t)=\frac{\left(2 a x_{3}-b x_{2} e^{2 \lambda t}\right) c}{2\left(x_{1} b^{2} e^{4 \lambda t}-x_{2} a b e^{2 \lambda t}+a^{2} x_{3}\right)}, \\
& v_{0}(t)=\frac{\left(2 b x_{1} e^{4 \lambda t}-a x_{2} e^{2 \lambda t}\right) c}{2\left(x_{1} b^{2} e^{4 \lambda t}-x_{2} a b e^{2 \lambda t}+a^{2} x_{3}\right)} .
\end{aligned}
$$

It can be seen that $v_{0}(t) \rightarrow 0$ as $t \rightarrow \infty$ at a rate of $e^{2 \lambda t}$. Now consider the "simplified" function

$$
\tilde{F}\left(t, w_{0}, v_{0}\right)=\left(\begin{array}{ll}
w & v
\end{array}\right)\left(\begin{array}{l}
w \\
v
\end{array}\right),
$$

which is minimized over the same constraint 4.51). The optimal sequence is now

$$
\begin{aligned}
& \tilde{w}_{0}(t)=\frac{a c}{a^{2}+b^{2} e^{4 \lambda t}}, \\
& \tilde{v}_{0}(t)=\frac{b c}{a^{2}+b^{2} e^{-4 \lambda t}},
\end{aligned}
$$

which also converges to the stable manifold, but at the rate of $e^{4 \lambda t}$. Because the "cross-term" of the quadratic is missing, the rate of convergence effectively doubles.

Of course, the faster convergence of the algorithm will be balanced by the computational cost of diagonalizing the Hamiltonian beforehand. Because eigenvector computation can be expensive, it may not be worthwhile to implement the modified problem for higher dimensional systems.

\section{EXAMPLES}

5.1. Example. Consider the $H_{\infty}$ problem for the nonlinear system

$$
\begin{aligned}
\dot{x} & =A x+\epsilon f(x)+B u, \\
y & =C x .
\end{aligned}
$$

Increasing $\epsilon$ is equivalent to strengthening the effect of the nonlinear terms on the system dynamics.

Define

$$
\begin{gathered}
A=\left(\begin{array}{cc}
0 & 1 \\
-1 & -2
\end{array}\right), \quad B=\left(\begin{array}{l}
0 \\
1
\end{array}\right), \quad C=\left(\begin{array}{ll}
1 & 0
\end{array}\right), \\
f(x)=\left(\begin{array}{c}
x_{1}^{2} \\
x_{1} x_{2}-x_{2}^{2}
\end{array}\right), \quad \gamma=1.3 .
\end{gathered}
$$

The algorithm was used to solve the corresponding Hamilton-Jacobi equation at the point $\left(\begin{array}{ll}0.1 & 0\end{array}\right)^{T}$ for different values of $\epsilon$. For all cases, the first terminal time in the sequence $t^{i}$ in (2.9a) used in the program was 2.0. Since the solution to the Hamilton-Jacobi equation is not known when $\epsilon \neq 0$, for all cases the simulation was run until five significant digits were obtained in the solution. The resulting state trajectories are given in Figure [5.1. This indicates that the algorithm is indeed finding a stabilizing trajectory. Figure 5.2 shows the error graph of the algorithm for each iteration for each value of $\epsilon$. 


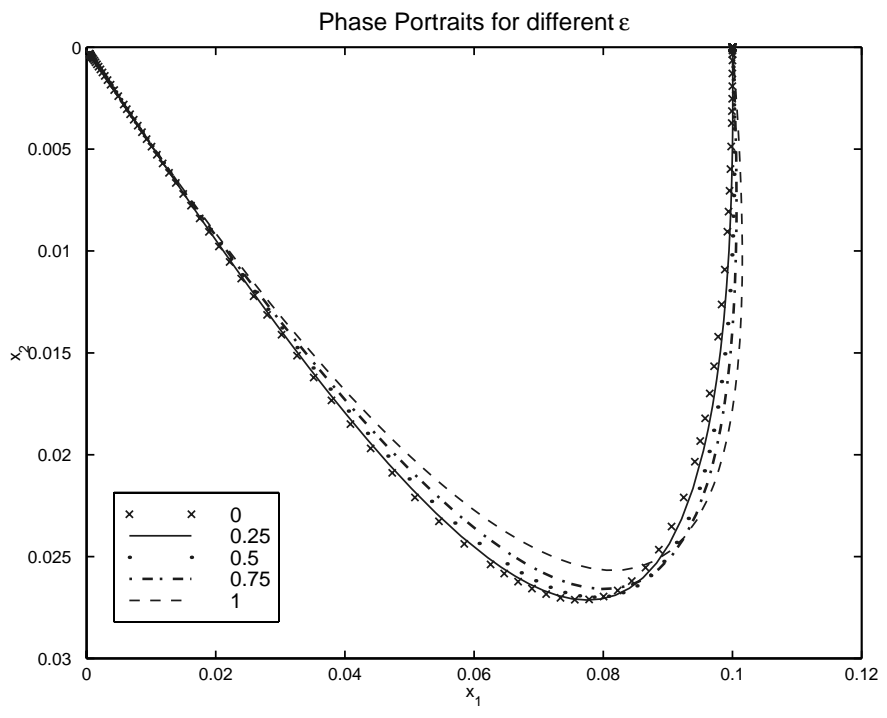

FIGURE 5.1. State trajectories of system with algorithmic control

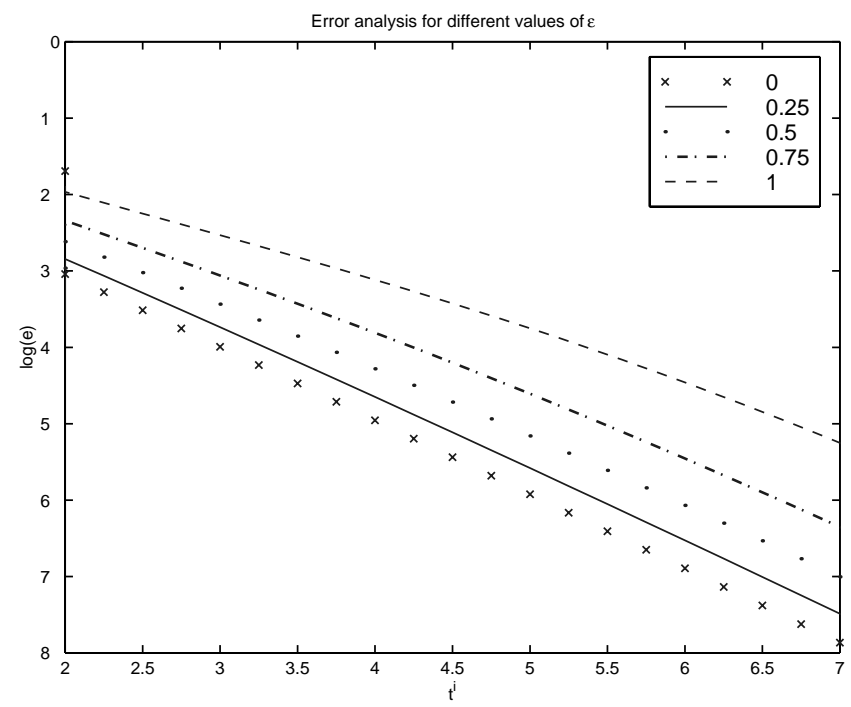

FIGURE 5.2. Effect of nonlinear terms on convergence

The eigenvalues of the corresponding Hamiltonian matrix are $\pm 1.31, \pm 0.48$. For the linear case $\epsilon=0$, the straight line of the error curve on the semi-logarithmic axis implies exponential convergence with respect to the iteration variable. The slope of the line indicates the rate of convergence, which can be calculated using a least square fit. In this case, the rate of convergence is -0.96 , which is indeed $O\left(e^{2 \lambda_{1} t}\right)$ as expected. As $\epsilon$ increases, the nonlinear terms have a stronger effect on the algorithm, and the rate of convergence decreases accordingly. 

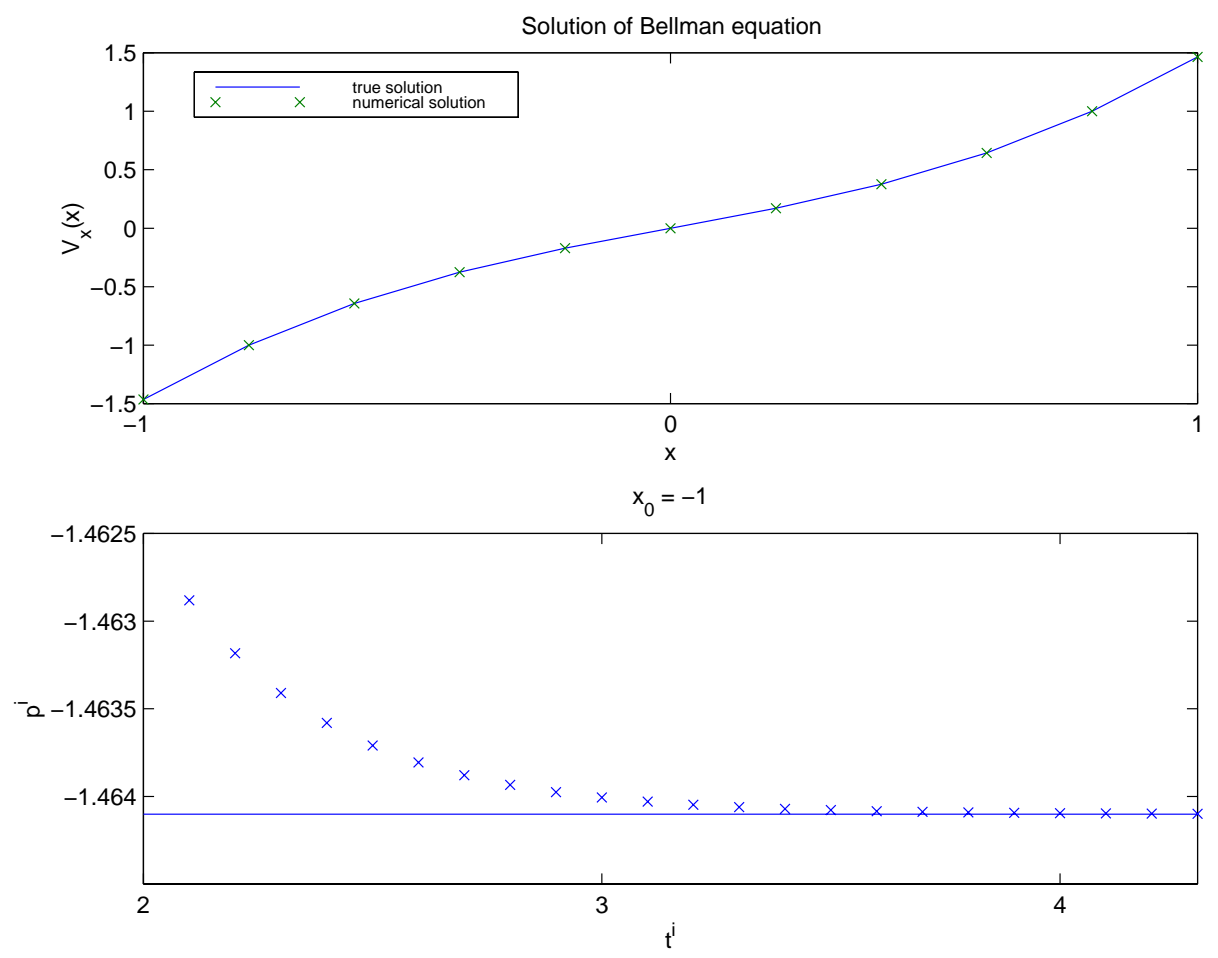

FiguRE 5.3. Numerical solution for system (5.1)

5.2. Optimal control problem with non-quadratic cost. The first example is a linear system with a non-quadratic cost function. The example is from [4]:

$$
\begin{aligned}
& \dot{x}=-a x+u, \\
& J=\int_{0}^{\infty}\left(x^{2}+x^{4}+u^{2}\right) d t .
\end{aligned}
$$

The corresponding Bellman equation is

$$
H\left(x, V_{x}\right)=-a x V_{x}-\frac{1}{4} V_{x}^{2}+x^{2}+x^{4}=0,
$$

and the optimal control of (5.1) is $u^{*}=-\frac{1}{2} V_{x}^{-}$, where

$$
V_{x}^{-}(x)=-2 x\left(a-\sqrt{a^{2}+1+x^{2}}\right) .
$$

In the case $a=1$, equation (5.2) was solved by the algorithm for $x \in[-1,1]$. The initial guess for $V_{x}^{-}(x)$ was chosen to be $-2 x$. The starting time in the sequence $t^{i}$ in (2.9a) was chosen as $t^{0}=2$, and the times were increased at each iteration by 0.1 .

The top graph of Figure 5.3 shows that the algorithm finds the stabilizing solution of (5.2) for different fixed values of $x$. The graph on the bottom shows the progress of the algorithm to the correct solution for $x=-1$, which is $V_{x}^{-}(-1)=$ -1.46410 . 

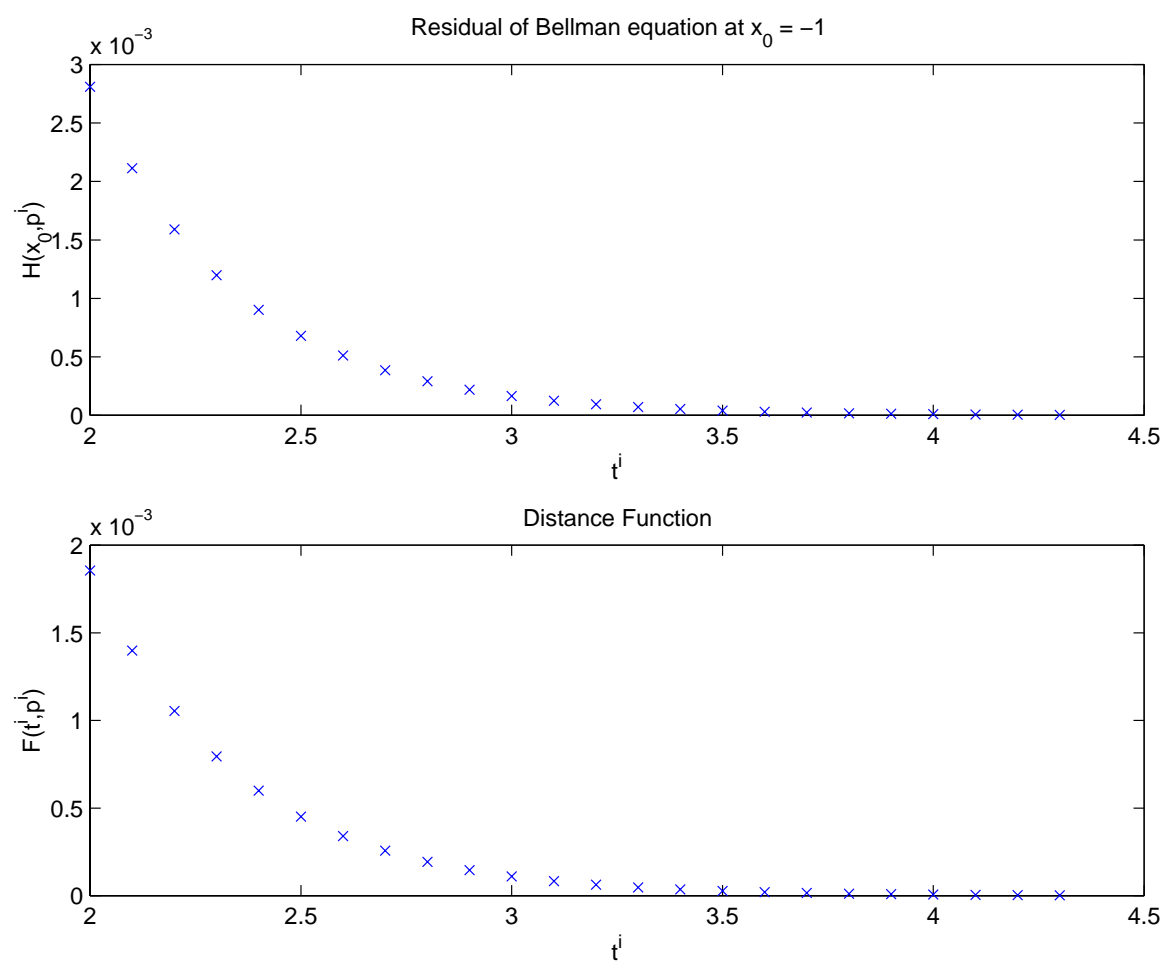

Figure 5.4. Error functions for system (5.1)

Figure 5.4 displays two error estimates used when the true solution is not known in advance. The graph on the top displays the first error estimate which is the residual of the Bellman equation for each minimizing initial condition $p_{0}^{i}$. Since the algorithm seeks a solution to the Bellman equation, each iteration should cause the residual to decrease to zero. The bottom graph shows the second error estimate which shows the progress of the distance function

$$
F\left(t^{i}, p_{0}^{i}\right)=\left\|x\left(t^{i}, x_{0}, p_{0}^{i}\right)\right\|^{2}+\left\|p\left(t^{i}, x_{0}, p^{i}\right)\right\|^{2}
$$

as $t^{i}$ increases. Clearly, this function should monotonically decrease to zero as well as $t^{i}$ grows bigger.

\subsection{A two-dimensional linear $H_{\infty}$ problem. For a linear system}

$$
\begin{aligned}
& \dot{x}=A x+B u, \\
& y=C x,
\end{aligned}
$$

the Hamilton-Jacobi-Isaacs equation, whose solution insures the $\mathcal{L}_{2}$ gain from $u$ to $y$ is less than or equal to $\gamma$, reduces to the Algebraic Ricatti Equation (2.10). Specifically, with $k=4 \gamma^{2}$, the stabilizing solution is $V_{x}^{-}=x^{T} P$, and the corresponding control is $u^{*}=\frac{1}{2 \gamma^{2}} B^{T}\left(V_{x}^{-}\right)^{T}=\frac{1}{2 \gamma^{2}} B^{T} P x$. 

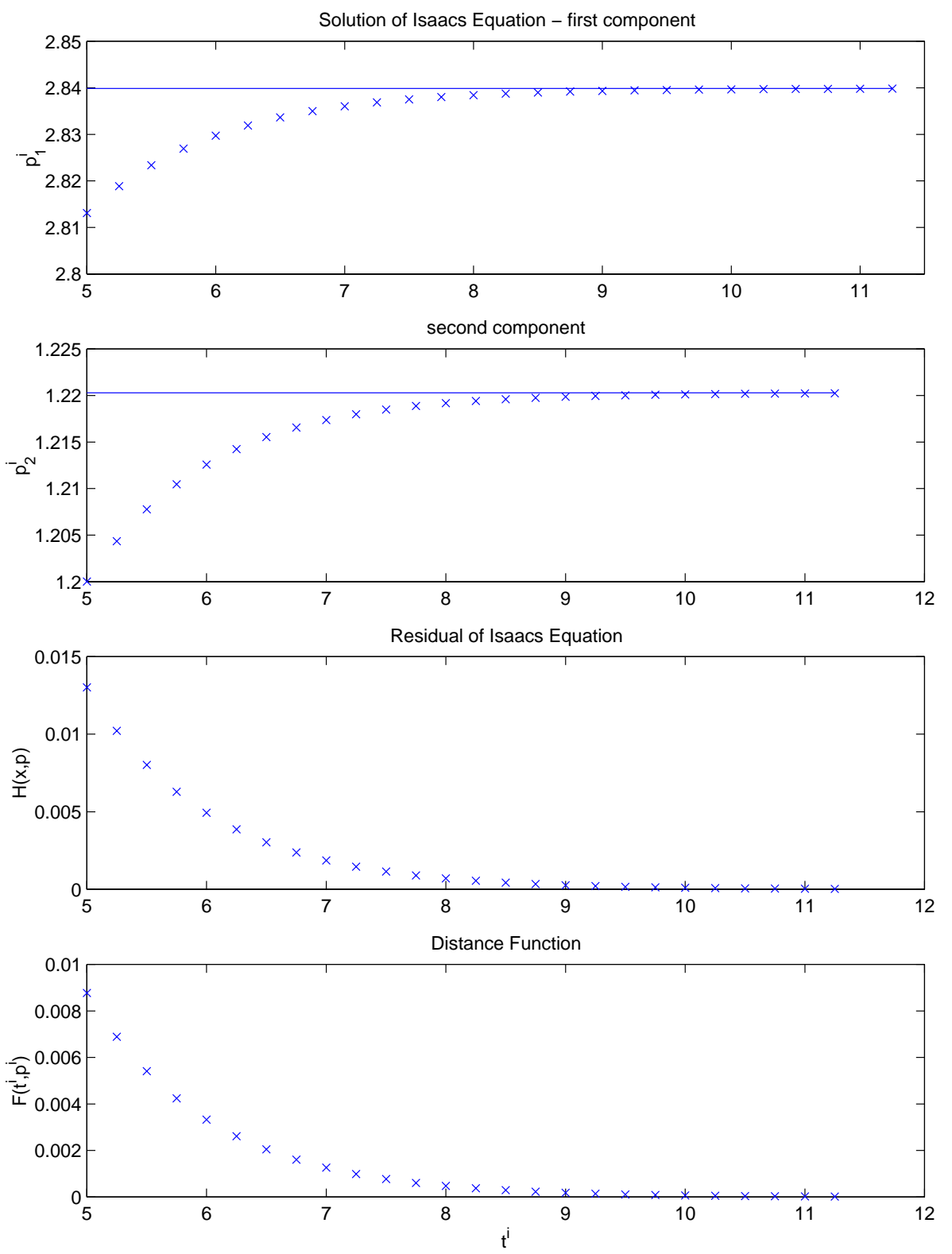

FiguRE 5.5. Solution and error analysis for equation (2.10)

Although (2.10) can be solved directly, the optimal control can also be found at a specific initial condition by the algorithm. Let

$$
\begin{aligned}
A & =\left(\begin{array}{cc}
0 & 1 \\
-1 & -2
\end{array}\right), \\
B & =\left(\begin{array}{l}
0 \\
1
\end{array}\right), \\
C & =\left(\begin{array}{ll}
1 & 0
\end{array}\right),
\end{aligned}
$$


and set $\gamma=1.3$. The algorithm was run at the initial point $x=\left(\begin{array}{ll}1 & 0\end{array}\right)^{T}$, with initial guess for $p^{*}$ arbitrarily chosen to be $\left(\begin{array}{ll}1 & 1\end{array}\right)^{T}$. The first time in the sequence $t^{i}$ in (2.9a) used in the program was $t^{0}=5$, and the times were increased at each iteration by 0.25 .

The true solution $V_{x}(x)=\left(\begin{array}{ll}2.839 & 1.220\end{array}\right)^{T}$ is found by directly solving the Algebraic Ricatti Equation. Figure 5.5 shows the convergence of each component of $p^{i}$ to the true solution. The bottom two graphs show the distance function $F$ and the residual function $H^{*}$, which indicate that the algorithm is indeed converging to a stabilizing solution of (2.10).

5.4. Convergence rate versus eigenvalues of Hamiltonian. Section 4.2 shows that for linear systems

$$
e^{i}=\left\|p_{0}^{i}-p_{0}^{*}\right\| \approx C e^{2 \operatorname{Re} \lambda_{1} t},
$$

where $\lambda_{1}$ is the eigenvalue with largest negative real part of $\bar{H}$. Therefore,

$$
\ln \left(e^{i}\right) \approx \ln C+2 \operatorname{Re} \lambda_{1} t^{i} .
$$

So if the graph of the error function versus the sequence $t^{i}$ is a straight line on semilog axes, then the error is truly decreasing exponentially as the times in the algorithm increase. Furthermore, the slope of the line will determine the time constant, or the rate of decay.

The Hamiltonian matrix associated with equation (5.2) is

$$
H=\left(\begin{array}{cc}
-a & -\frac{1}{2} \\
-2 & a
\end{array}\right) \text {. }
$$

$H$ has eigenvalues $\pm \sqrt{a^{2}+1}$. Running the algorithm at $x=1$ for different values of $a$, Figure [5.6 displays the logarithmic error graph. Clearly, the curves are all linear, which imply exponential convergence. Furthermore, as a increases,

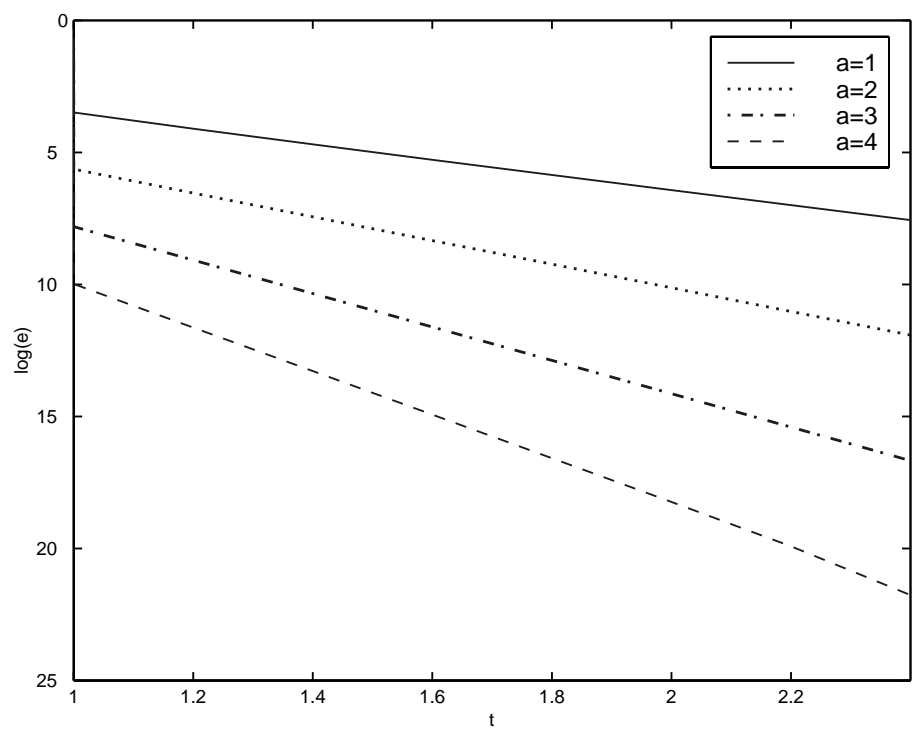

FiguRE 5.6. Error at each iteration for different $a$ 
TABLE 5.1. Eigenvalues of $H$ vs. rate of convergence

\begin{tabular}{|c|c|c|c|}
\hline $\mathrm{a}$ & $\lambda$ & $2 \lambda$ & $m_{\text {calc }}$ \\
\hline 1 & $\sqrt{2}$ & -2.83 & -2.83 \\
\hline 2 & $\sqrt{5}$ & -4.47 & -4.46 \\
\hline 3 & $\sqrt{10}$ & -6.32 & -6.37 \\
\hline 4 & $\sqrt{17}$ & -8.24 & -8.25 \\
\hline
\end{tabular}

the slope of each line increases, indicating a faster rate of convergence. By finding the least square fit to each curve, the rate of convergence can be tested. Table 5.1 shows how the eigenvalues of the Hamiltonian and the convergence rate of the algorithm vary as $a$ increases. Clearly, the rate of convergence is consistently twice the eigenvalue of the Hamiltonian matrix.

For higher dimensional systems, this convergence rate depends on the eigenvalue with largest negative real part eigenvalue of the Hamiltonian. Consider the linear $H_{\infty}$ problem (5.4), (5.5). The associated Hamiltonian system of the Isaacs equation is

$$
\left(\begin{array}{c}
\dot{x} \\
\dot{p}
\end{array}\right)=\left(\begin{array}{cc}
A & \frac{1}{2 \gamma^{2}} B B^{T} \\
-2 C^{T} C & -A^{T}
\end{array}\right)\left(\begin{array}{l}
x \\
p
\end{array}\right)
$$

and the eigenvalues of (5.9) are $\pm 1.31, \pm 0.48$. Figure 5.7 shows the graph of the error versus the time on semilog axes. The straight line indicates that the convergence is indeed exponential, and the slope confirms that the rate is approximately double the minimum eigenvalue of $H$.

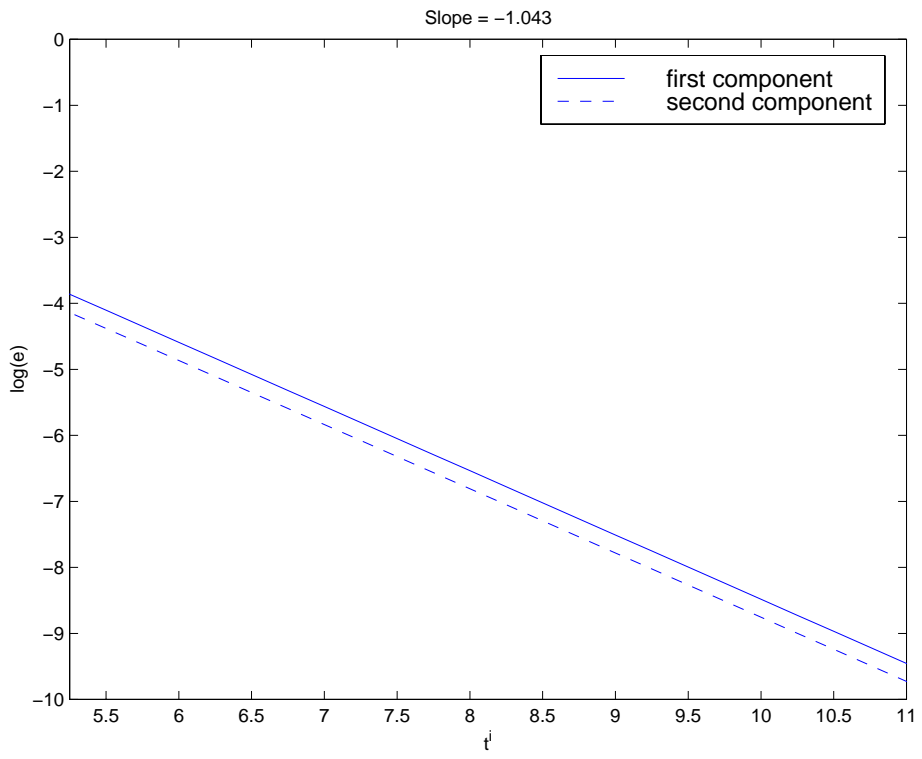

Figure 5.7. Convergence rate of algorithm for system (5.5) 


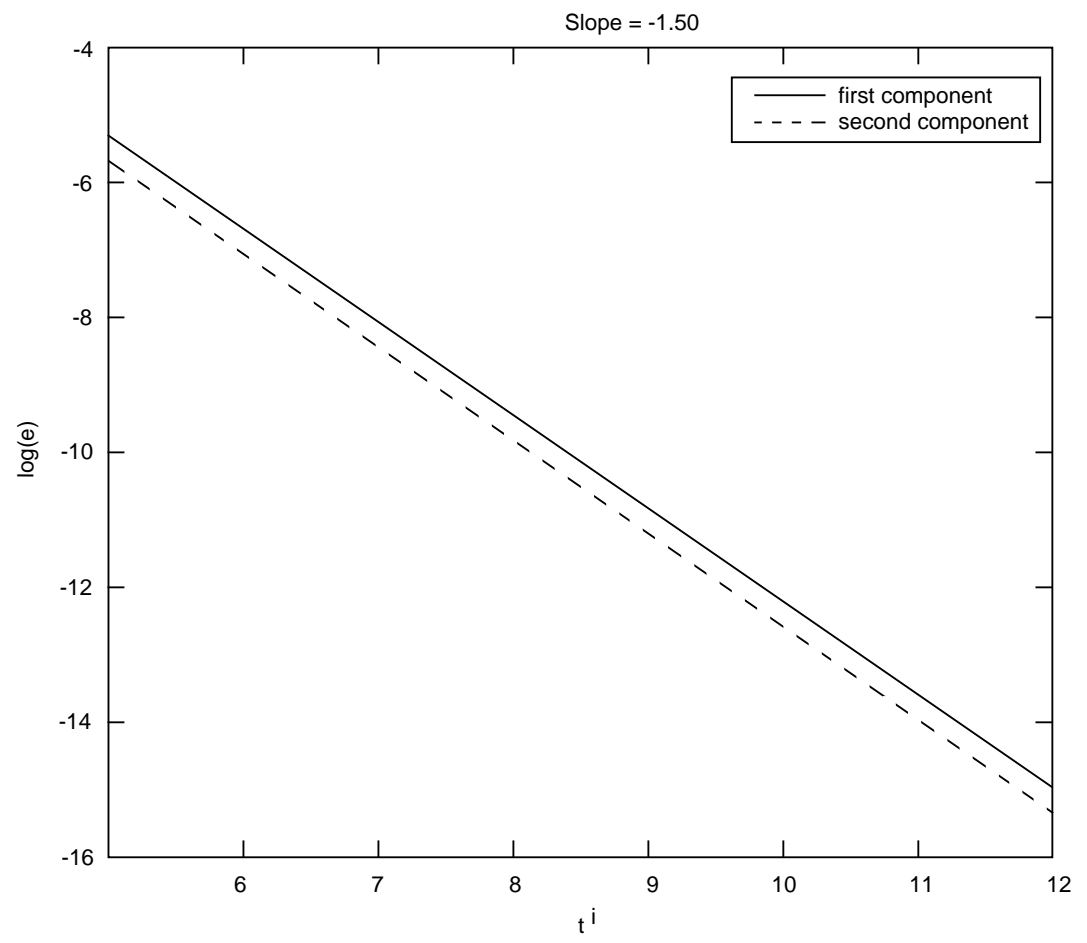

FiguRE 5.8. Convergence rate when $\gamma=2$

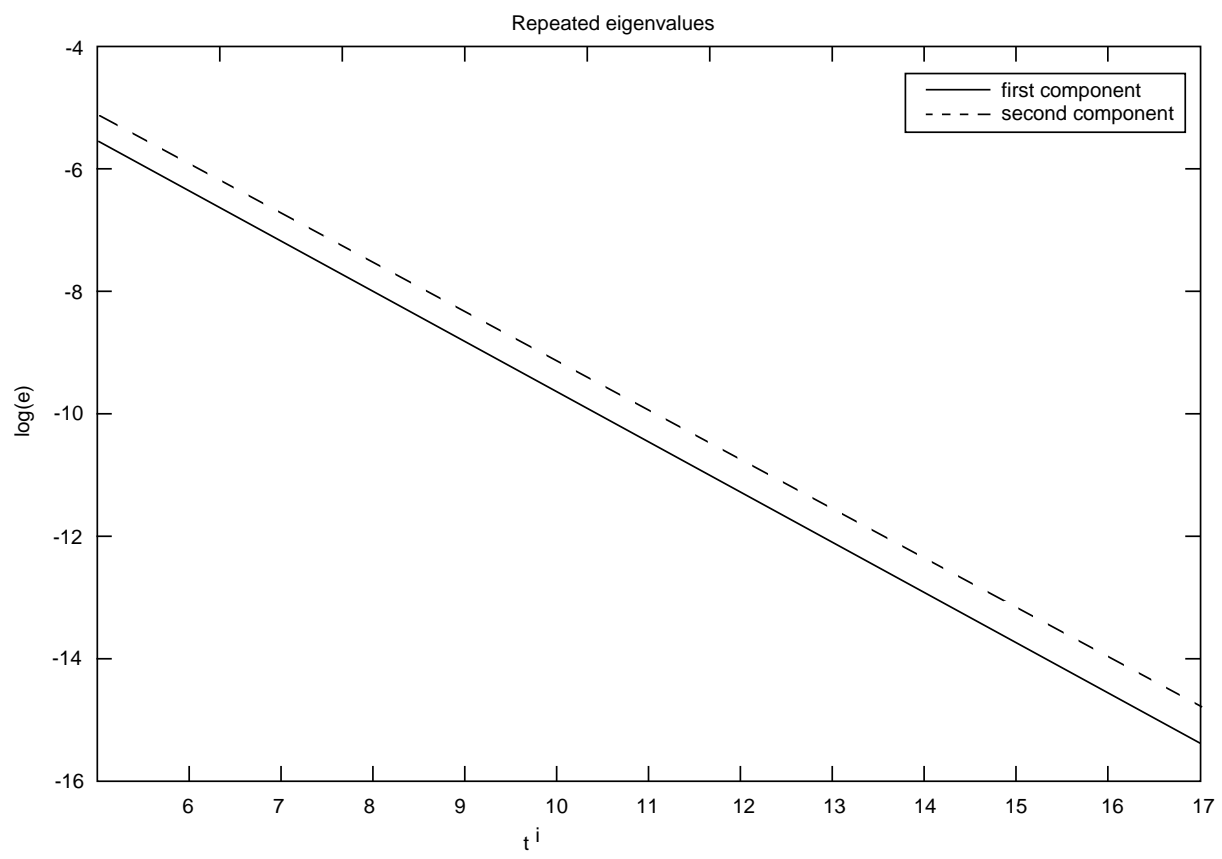

FiguRE 5.9. Error graph for repeated eigenvalues 
By changing the $H_{\infty}$-gain to $\gamma=2$, the eigenvalues of the Hamiltonian change to $\pm 0.721, \pm 1.20$. Correspondingly, Figure 5.8 shows a faster convergence rate of -1.50 .

Section 4 implies that as the eigenvalues of the Hamiltonian matrix move toward the imaginary axis, the algorithm takes longer to converge to the stabilizing solution. This corresponds to the fact that as $\sigma(H)$ moves closer to zero, the $H_{\infty}$ problem approaches the singular case.

Next, the case where $H$ has repeated eigenvalues is considered. Suppose $H$ has eigenvalues $\left\{\frac{1}{2}, \frac{1}{2},-\frac{1}{2},-\frac{1}{2}\right\}$. The theoretical convergence rate is of the order $t e^{-.5 t}$. Figure 5.9 shows the logarithmic error graph.

While the resulting graphs are not linear, a least square fit of the data to a straight line gives a slope of -0.8376 , which is less than twice the smallest eigenvalue. Therefore, just as repeated eigenvalues slow the rate of a stable system to the equilibrium point, they also slow the convergence of the algorithm to the stable manifold.

\section{ACKNOWLEDGMENT}

The careful reading of this paper and the helpful comments are appreciated. In particular one of the reviewers pointed out the need for assumption 2. Another reviewer cited the work in 2] and in [14].

\section{REFERENCES}

[1] Arthur Bryson, and Yu-Chi Ho, Applied optimal control; optimization, estimation, and control, Blaisdell Pub. Co., 1969, Waltham, MA. MR 56:4953 (rev. printing)

[2] Hans Knobloch, Alberto Isidori, and Dietrich Flockerzi, Topics in Control Theory, Birkhauser Verlag, 1993. MR 95e:93002

[3] W.L. Garrard, Additional results on suboptimal feedback control for nonlinear systems, International Journal of Control, 1969, 10(6), 657-663. MR 41:5084

[4] Y. Nishikawa, A method for suboptimal design of nonlinear feedback systems, Automatica, 1971, 7, 703-712. MR 48:2860

[5] G.N. Saridis, and C.-S.G. Lee, An approximation theory of optimal control for trainable manipulators, IEEE Transactions on Systems, Man. and Cybernetics, 1979, SMC-9(3), 152159. MR 80b:93070

[6] Randal Beard, George Saridis, and John Wen, Improving the Performance of Stabilizing Controls for Nonlinear Systems, IEEE Journal on Control Systems, 1996, 27-35.

[7] K.A. Wise, and J.L. Sedwick, Missile autopilot design using nonlinear $H_{\infty}$-optimal control, Proceedings of 13th IFAC Symposium on Automatic Control in Aerospace, 1994.

[8] Jerry Markman, Numerical Solutions of the Hamilton-Jacobi Equations Arising in Nonlinear $H_{\infty}$ and Optimal Control, Washington University, Department of Systems Science and Mathematics, D.Sc. thesis 1998.

[9] Jerry Markman, and I. Norman Katz, An Iterative Algorithm for Solving Hamilton-Jacobi Equations, SIAM J. Sci. Comput. 22 (2000), 312-329. CMP 2000:15

[10] Alberto Isidori, Attenuation of Disturbances in Nonlinear Control Systems, Systems, Models and Feedback, A. Isidori, and T.J. Tarn, editors, 1992, Birkhauser, pages 275-300. MR 93f:93047

[11] W. A. Coppel, Stability and Asymptotic Behavior of Differential Equations, D.C. Heath and Company, 1965, Boston.

[12] S. Wiggins, Introduction to Applied Nonlinear Dynamical Systems and Chaos, SpringerVerlag, 1990, New York. MR 92a:58041

[13] Richard Bellman, Stability Theory of Differential Equations, McGraw-Hill, 1953, New York. MR 15:794b 
[14] A. J. van der Schaft, $L_{2}$-Gain Analysis of Nonlinear Systems and Nonlinear State Feedback $H_{\infty}$ Control, IEEE Trans. on Automatic Control, 37, 1992, 770-784. MR 93e:93027]

[15] A. M. Stuart and A. R. Humphries, Dynamical Systems and Numerical Analysis, Cambridge University Press, 1996. MR 97g:65009

Department of Systems Science and Mathematics, Washington University, Campus Box 1040, One Brookings Drive, St. Louis, Missouri 63130

E-mail address: jerry@zach.wustl.edu

Department of Systems Science and Mathematics, Washington University, Campus Box 1040, One Brookings Drive, St. Louis, Missouri 63130

E-mail address: katz@zach.wustl.edu 